\title{
elsevier_STOTEN_17069
}

\section{Isotopic and hydrogeochemical characterization of high-altitude karst aquifers in complex geological settings. The Ordesa and Monte Perdido National Park (Northern Spain) case study}

\section{L.J.L.J. Lambán ${ }^{a}$}

javier.lamban@igme.es

J. Jódar ${ }^{b}$

jorge.jodar@upc.edu

E. Custodio

emilio.custodio@upc.edu

A. Soler

albertsoler@ub.edu

G. Sapriza ${ }^{d}$

g.sapriza@usask.ca

R. Soto ${ }^{a}$

r.soto@igme.es

${ }^{\mathrm{a} G e o l o g i c a l}$ Survey of Spain (IGME), Spain

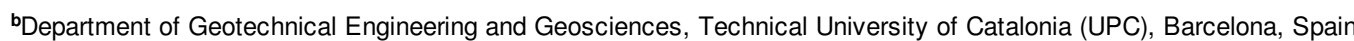

'Grup de Mineralogia Aplicada i Medi Ambient, Departament Cristal lografia Mineralogia i Dipòsits Minerals, Facultat de Geologia, Universitat de Barcelona (UB), Spain

${ }^{d}$ Global Institute for Water Security, National Hydrology Research Centre, Canada

Editor: D. Barcelo

\section{Abstract}

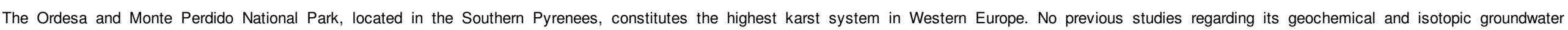

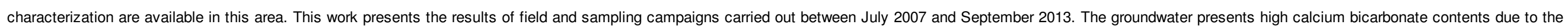

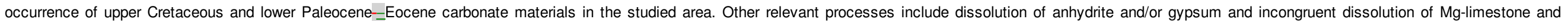

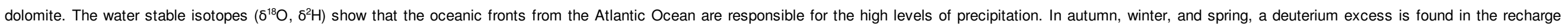

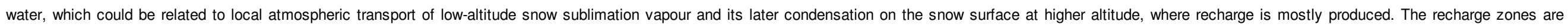

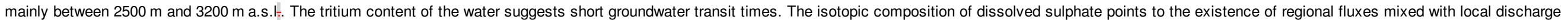

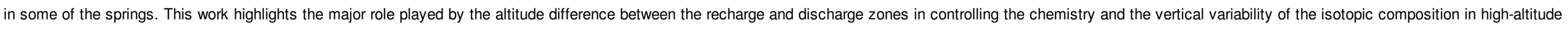
karst aquifers.

Keywords: Environmental isotopes; Snow sublimation; Deuterium excess; Karst hydrology; Alpine hydrology; Parque Nacional Ordesa y Monte Perdido

\section{Introduction}




\section{elsevier_STOTEN_17069}

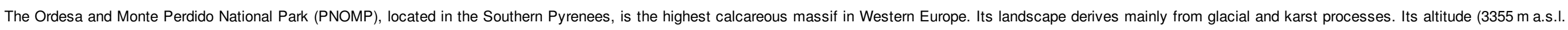

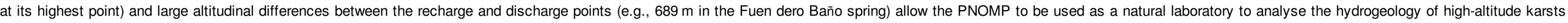
and how the isotopic and hydrogeochemical features of groundwater vary with altitude.

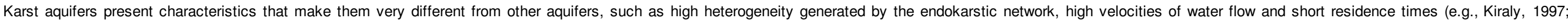

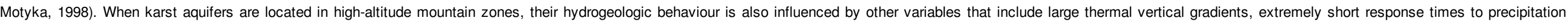

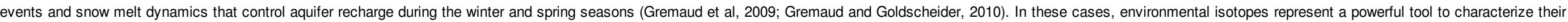

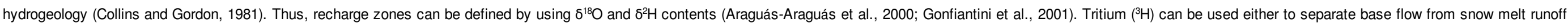

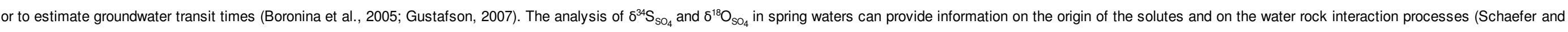
Usdowski, 1992; González-Fernández et al., 2009).

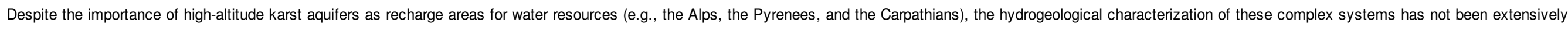

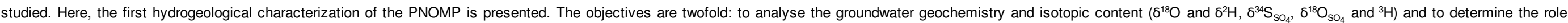
played by the high altitude and low temperature conditions in the hydrogeological behaviour of karst systems.

\section{The study area}

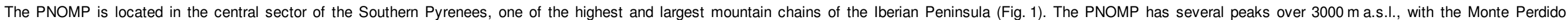

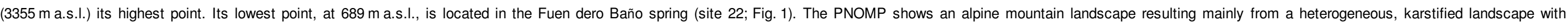
numerous karren fields, caves, sinkholes and fissures and from glacial processes as shown by the long and deep, U-shaped, glacial valleys of Ordesa, Añisclo, Escuaín and Pineta (Fig. 1). 


\section{elsevier_STOTEN_17069}
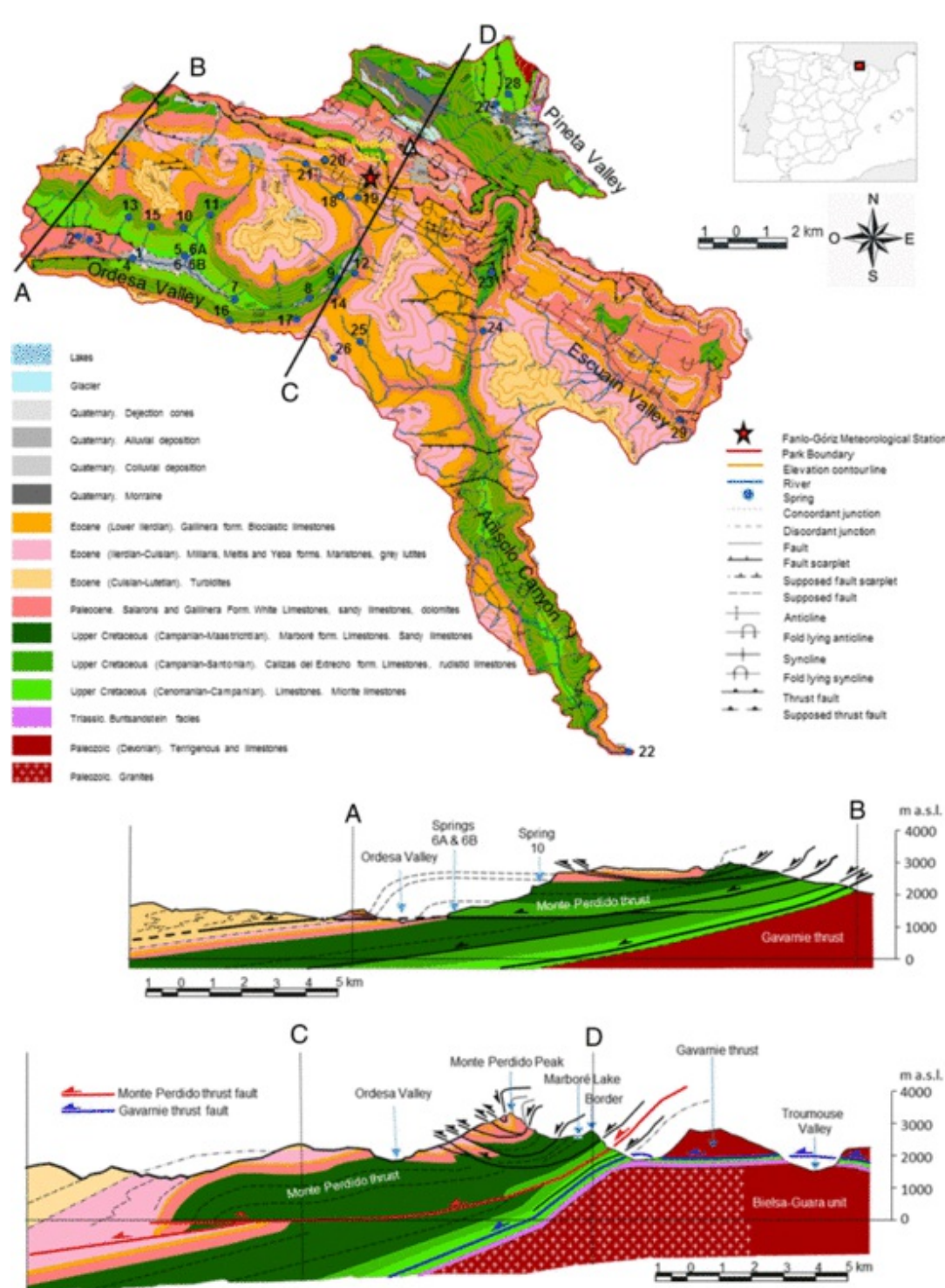

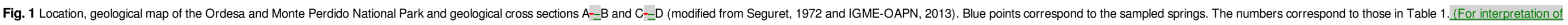
the references to colour in this figure legend, the reader is referred to the web version of this article.)

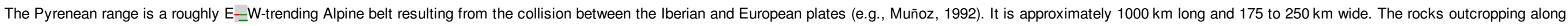

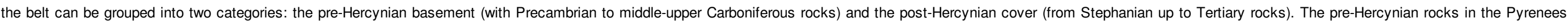

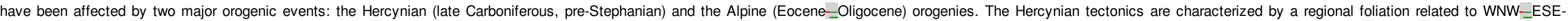

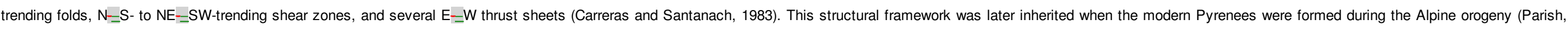

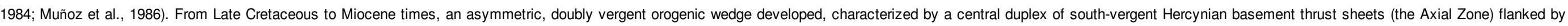
north- and south-vergent fold and thrust belts to the north and south, respectively, and the associated foreland basins (e.g., Muñoz, 1992). 


\section{elsevier_STOTEN_17069}

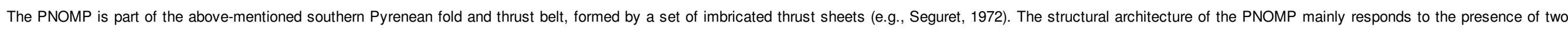

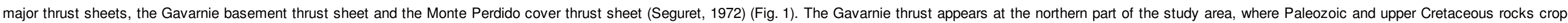

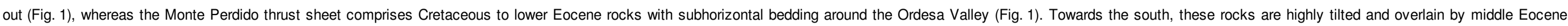

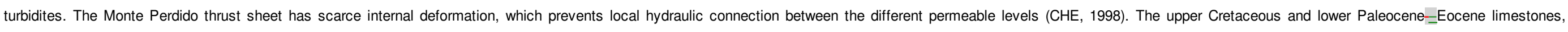

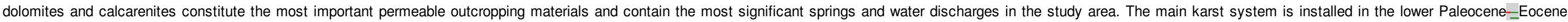
materials (CHE, 1998; Ríos-Aragüés, 2003).

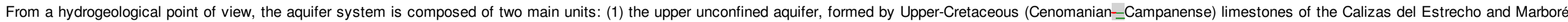

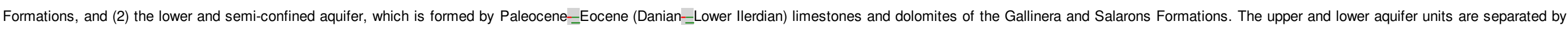
the less permeable terrigenous facies of the Maestrichtian.

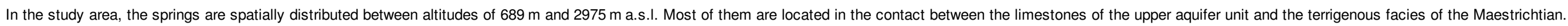

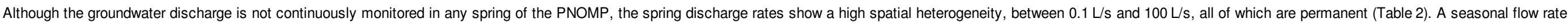
variation has also been observed in the sampled springs, in agreement with the fast reaction to rainfall of spring discharge in such a developed karst system (Ríos-Aragüés, 2003; García-Ruiz et al., 2014).

Table 1 Atmospheric circulation pattern classification.

\begin{tabular}{|c|c|}
\hline ACP type & Description \\
\hline N/NW/W/SW & Northerly/northwesterly/westerly/southwesterly directional types \\
\hline $\mathrm{NE} / \mathrm{E} / \mathrm{SW} / \mathrm{S}$ & Northeasterly/easterly/southeasterly/southerly directional types \\
\hline C & Cyclonic \\
\hline UC & Unclassified/light flow-cyclonic \\
\hline HYC & Hybrid-cyclonic \\
\hline A & Anticyclonic \\
\hline UA & Unclassified/light flow-anticyclonic \\
\hline HYA & Hybrid-anticyclonic \\
\hline
\end{tabular}

Table 2 Groundwater sampling points (springs) and conducted field campaigns.

\begin{tabular}{|c|c|c|c|c|c|c|c|c|c|}
\hline \multirow[t]{2}{*}{ Code } & \multirow[t]{2}{*}{ Name } & \multirow{2}{*}{$\begin{array}{l}\text { Discharge height } \\
\text { (m a.s.I.) }\end{array}$} & \multirow{2}{*}{$\begin{array}{l}\text { Recharge height (a) } \\
\text { (m a.s.l.) }\end{array}$} & \multirow{2}{*}{$\underset{(\mathrm{m})}{\Delta \mathrm{Z}-\mathrm{b})}$} & \multirow[t]{2}{*}{ Sector } & \multicolumn{4}{|c|}{ Field campaigns } \\
\hline & & & & & & $\left.2007 f^{c}\right)$ & $2011\left(^{d}\right)$ & $2012\left(f^{d}\right)$ & $\left.2013^{(e)}\right)$ \\
\hline 1 & Arazas River & 1130 & 3142 & 2012 & Ordesa & Jul & $=$ & $=$ & $=$ \\
\hline 2 & Fuen Plana Canal & 1280 & 2815 & 1535 & Ordesa & Jul & $=$ & $=$ & $=$ \\
\hline 3 & Fuen Caño & 1290 & 2978 & 1688 & Ordesa & Jul & $=$ & $=$ & $=$ \\
\hline 4 & As Fuens & 1349 & 3025 & 1676 & Ordesa & $=$ & May, Jun, Jul, Aug, Sep, Oct, Nov, Dec & Mar, Apr & $=$ \\
\hline 5 & Fuen Roya=_Aguas_Abajo & 1348 & 3108 & 1760 & Ordesa & Jul & $=$ & $=$ & $=$ \\
\hline 6 & Fuen Roya_-Surgencia & 1358 & 3108 & 1750 & Ordesa & Jul & $=$ & $=$ & $=$ \\
\hline $6 \mathrm{~A}$ & Fuen Roya-1 & 1358 & 3319 & 1971 & Ordesa & $=$ & $=$ & $=$ & Sep \\
\hline
\end{tabular}




\section{elsevier_STOTEN_17069}

\begin{tabular}{|c|c|c|c|c|c|c|c|c|c|}
\hline $6 B$ & Fuen Roya-2 & 1358 & 3091 & 1743 & Ordesa & $=$ & $=$ & $=$ & Sep \\
\hline 7 & Fuen d-Arripas & 1440 & 2923 & 1483 & Ordesa & Jul & $=$ & $=$ & $=$ \\
\hline 8 & Fuen as Gradas & 1700 & 2915 & 1215 & Ordesa & Jul & $=$ & $=$ & $=$ \\
\hline 9 & Fuen Mochera & 1807 & 3185 & 1435 & Ordesa & Jul & Sep, Oct, Nov, Dec & Feb, Mar, Apr & $=$ \\
\hline 10 & Fuen linabellá & 1750 & 3197 & 1447 & Ordesa & Jul & $=$ & $=$ & Sep \\
\hline 11 & Fuen Cotatuero & 1800 & 3067 & 1267 & Ordesa & Jul & $=$ & $=$ & $=$ \\
\hline 12 & Fuen De Soaso-_Torrente & 1820 & 2739 & 919 & Ordesa & Jul & $=$ & $=$ & $=$ \\
\hline 13 & Fuen Carriata & 1820 & 2763 & 943 & Ordesa & Jul & $=$ & $=$ & $=$ \\
\hline 14 & Fuen De Soaso & 1830 & 2574 & 744 & Ordesa & Jul & $=$ & $=$ & $=$ \\
\hline 15 & Fuen Gallinero & 1840 & 3041 & 1201 & Ordesa & Jul & $=$ & $=$ & $=$ \\
\hline 16 & Fuen Freda & 1860 & 2913 & 1053 & Ordesa & Jul & $=$ & $=$ & $=$ \\
\hline 17 & Fuen de L'Abe & 1920 & 2614 & 694 & Ordesa & Jul & $=$ & $=$ & $=$ \\
\hline 18 & Fuen Roldán & 2136 & 2855 & 719 & Ordesa & $=$ & Aug, Sep, Oct, Nov & Mar & $=$ \\
\hline 19 & Fuen de Goriz & 2200 & 3062 & 862 & Ordesa & Jul & May, Jun, Jul, Aug, Sep, Oct, Dec & Mar & $=$ \\
\hline 20 & Fuen Fria & 2340 & 3074 & 734 & Ordesa & Jul & $=$ & $=$ & $=$ \\
\hline 21 & Collado Millares & 2420 & 3120 & 700 & Ordesa & Jul & $=$ & $=$ & $=$ \\
\hline 22 & Fuen dero Baño & 689 & 2794 & 2105 & Añisclo & Jul & $=$ & $=$ & Sep \\
\hline 23 & Font Blanca & 1710 & 3099 & 1389 & Añisclo & Jul & May Jun, Jul, Sep, Oct, Nov & Mar & $=$ \\
\hline 24 & Fuen dero Foratiello & 1790 & 2670 & 880 & Añisclo & Jul & $=$ & $=$ & $=$ \\
\hline 25 & Fuen dero Furicón & 2010 & 2753 & 743 & Añisclo & Jul & $=$ & $=$ & $=$ \\
\hline 26 & Fuen dero Esmoladera & 2050 & 2725 & 675 & Añisclo & Jul & $=$ & $=$ & $=$ \\
\hline 27 & Fuen el Felcarral & 1703 & 3076 & 1373 & Pineta & Jul & $=$ & $=$ & $=$ \\
\hline 28 & Fuen de la Bispeta & 1930 & 2873 & 943 & Pineta & Jul & $=$ & $=$ & $=$ \\
\hline 29 & Fuen de Escuaín & 1050 & 2500 & 1450 & Escuaín & Jul & May, Jun, Jul, Aug, Sep, Oct, Nov & Mar, Apr & $=$ \\
\hline
\end{tabular}

a Recharge level obtained from the local altitudinal line,

${ }^{\mathrm{b}}$ Difference between the recharge and discharge levels.

${ }^{c}$ Major ions, $\delta^{2} \mathrm{H}, \delta^{18} \mathrm{O}$ and tritium.

d Major ions, $\delta^{2} \mathrm{H}$ and $\delta^{18} \mathrm{O}$,

e Major ions, $\delta^{2} \mathrm{H}, \delta^{18} \mathrm{O}, \delta^{34} \mathrm{~S}_{\mathrm{SO}_{4}}$ and $\delta^{18} \mathrm{O}_{\mathrm{SO}_{4} \text { - }}$

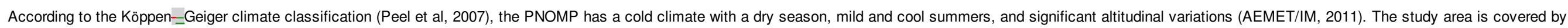

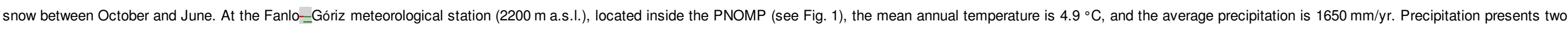
peaks in spring and autumn, at 220 and $185 \mathrm{~mm} / \mathrm{month}$, respectively, and two minima in winter and summer, at 80 and $105 \mathrm{~mm} / \mathrm{month}$, respectively (Fig. 2A). 


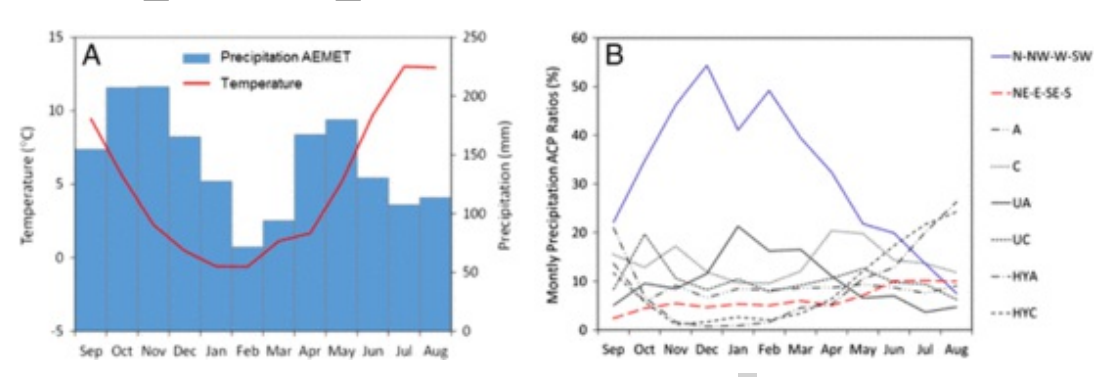

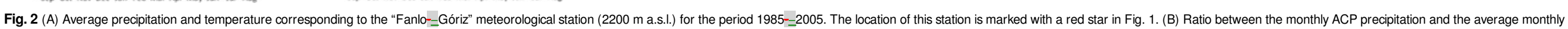

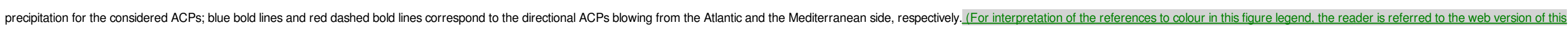
article.)

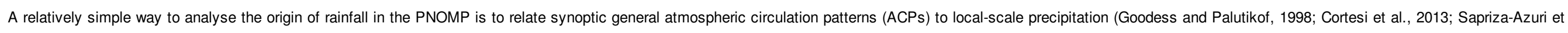

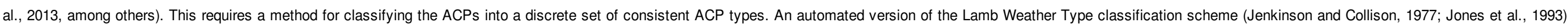

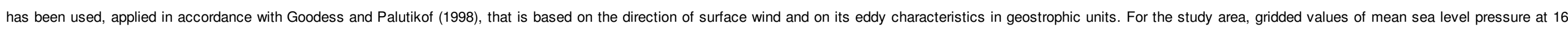

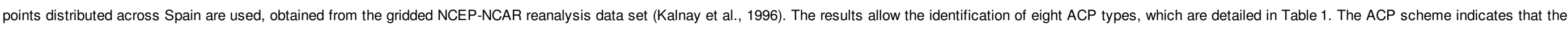

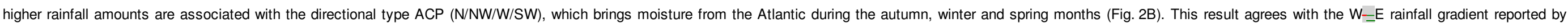

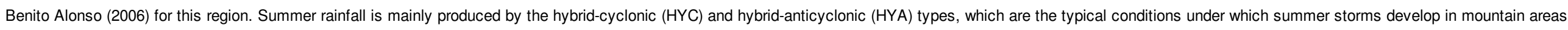

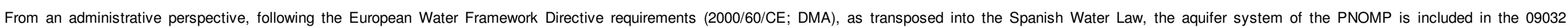
groundwater body named Sierra Tendeñera_Monte Perdido by the Ebro-s Hydrographic Confederation (CHE), which is the water authority.

\section{Materials and methods}

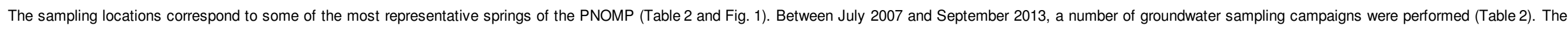

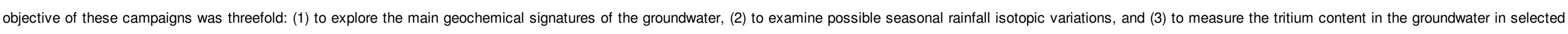
springs (Fig. 1 and Table 3).

Table 3 Representative physical, chemical and isotopic data of groundwater in the study area during the field campaign conducted in 2007.

\begin{tabular}{|c|c|c|c|c|c|c|c|c|c|c|c|c|c|c|c|c|c|c|}
\hline Sampling point code & Date & $T\left(f^{a}\right)$ & $\mathrm{pH}^{(a)}$ & $E C\left(x^{2}\right)$ & $\mathrm{DQO}$ & $\mathrm{HCO}_{3}$ & $\mathrm{SO}_{4}$ & $\mathrm{Cl}$ & $\mathrm{NO}_{3}$ & $\mathrm{Na}$ & $\mathrm{K}$ & $\mathrm{Ca}$ & $\mathrm{Mg}$ & $\mathrm{SIO}_{2}$ & $\delta^{2} \mathrm{H}$ & $\delta^{18} \mathrm{O}$ & $d\left(p^{b}\right)$ & ${ }^{3} \mathrm{H} \pm \mathrm{O}$ \\
\hline 1 & 20/07/2007 & 10.5 & 8.15 & 197 & 0.5 & 83.0 & 22.0 & 1.0 & 1.0 & 0.0 & 0.0 & 26.0 & 8.0 & 1.3 & $=74.9$ & $=10.7$ & 10.7 & $=$ \\
\hline 2 & 20/07/2007 & 10.9 & 8.29 & 302 & 0.5 & 162.0 & 12.0 & 1.0 & 1.0 & 0.0 & 0.0 & 37.0 & 15.0 & 4.6 & $=68.0$ & $=9.5$ & 8.3 & $6.08 \pm 0.62$ \\
\hline 3 & 20/07/2007 & 9.6 & 7.98 & 388 & 0.6 & 163.0 & 47.0 & 1.0 & 1.0 & 0.0 & 0.0 & 38.0 & 24.0 & 4.5 & $=71.2$ & $=10.2$ & 10.0 & $5.72 \pm 0.61$ \\
\hline 5 & 19/07/2007 & 10.5 & 7.89 & 930 & 0.5 & 103.0 & 380.0 & 3.0 & 0.0 & 2.0 & 0.0 & 136.0 & 40.0 & 5.7 & $=75.7$ & $=11.0$ & 11.9 & $4.89 \pm 0.59$ \\
\hline 6 & 19/07/2007 & 9.3 & 7.75 & 405 & 0.5 & 102.0 & 100.0 & 2.0 & 1.0 & 0.0 & 0.0 & 52.0 & 17.0 & 3.1 & $=75.7$ & $=11.0$ & 11.9 & $4.89 \pm 0.59$ \\
\hline 7 & 20/07/2007 & 11.6 & 8.11 & 306 & 0.7 & 149.0 & 26.0 & 1.0 & 0.0 & 0.0 & 0.0 & 40.0 & 14.0 & 3.2 & $=69.7$ & $=10.0$ & 10.2 & $4.87 \pm 0.59$ \\
\hline 8 & 18/07/2007 & 7.5 & 8.13 & 304 & 0.5 & 133.0 & 48.0 & 1.0 & 0.0 & 0.0 & 0.0 & 34.0 & 20.0 & 4.8 & $=69.3$ & $=10.0$ & 10.7 & $4.93 \pm 0.57$ \\
\hline 9 & 18/07/2007 & 7.0 & 7.94 & 92 & 0.5 & 61.0 & 13.0 & 1.0 & 0.0 & 0.0 & 0.0 & 14.0 & 8.0 & 0.3 & $=75.9$ & $=10.9$ & 10.9 & $4.98 \pm 0.57$ \\
\hline 10 & 19/07/2007 & 11.4 & 8.04 & 361 & 0.5 & 95.0 & 86.0 & 1.0 & 1.0 & 0.0 & 0.0 & 35.0 & 22.0 & 5.4 & $=75.7$ & $=11.0$ & 11.9 & $4.89 \pm 0.59$ \\
\hline 11 & 19/07/2007 & 10.0 & 8.11 & 299 & 0.5 & 108.0 & 40.0 & 2.0 & 1.0 & 0.0 & 0.0 & 33.0 & 14.0 & 4.0 & $=72.7$ & $=10.5$ & 11.5 & $5.46 \pm 0.61$ \\
\hline
\end{tabular}




\section{elsevier_STOTEN_17069}

\begin{tabular}{|c|c|c|c|c|c|c|c|c|c|c|c|c|c|c|c|c|c|c|}
\hline 12 & 20/07/2007 & $=$ & 8.33 & 244 & 0.6 & 124.0 & 16.0 & 1.0 & 0.0 & 0.0 & 0.0 & 37.0 & 8.0 & 2.6 & $=64.6$ & $=9.5$ & 11.3 & $=$ \\
\hline 13 & 19/07/2007 & 16.4 & 8.13 & 221 & 0.6 & 111.0 & 19.0 & 1.0 & 0.0 & 0.0 & 0.0 & 33.0 & 8.0 & 2.6 & $=66.6$ & $=9.4$ & 8.5 & $=$ \\
\hline 14 & 20/07/2007 & $=$ & 6.87 & 434 & 2.5 & 232.0 & 8.0 & 1.0 & 0.0 & 0.0 & 0.0 & 69.0 & 9.0 & 1.5 & $=61.2$ & $=8.9$ & 10.1 & $=$ \\
\hline 15 & 19/07/2007 & 12.1 & 8.29 & 274 & 0.6 & 106.0 & 33.0 & 2.0 & 0.0 & 0.0 & 0.0 & 34.0 & 11.0 & 3.0 & $=71.9$ & $=10.5$ & 11.7 & $4.55 \pm 0.58$ \\
\hline 16 & 20/07/2007 & 7.9 & 8.25 & 237 & 0.6 & 125.0 & 12.0 & 1.0 & 0.0 & 0.0 & 0.0 & 24.0 & 15.0 & 2.3 & $=68.7$ & $=10.1$ & 11.8 & $=$ \\
\hline 18 & $20 / 07 / 2007$ & 8.4 & 8.16 & 210 & 0.5 & 102.0 & 12.0 & 1.0 & 2.0 & 0.0 & 0.0 & 30.0 & 7.0 & 1.5 & $=63.1$ & $=8.9$ & 8.2 & $5.76 \pm 0.62$ \\
\hline 19 & $18 / 07 / 2007$ & 11.5 & 8.19 & 120 & 0.5 & 57.0 & 13.0 & 1.0 & 1.0 & 0.0 & 0.0 & 18.0 & 5.0 & 0.5 & $=72.9$ & $=10.5$ & 10.7 & $4.82 \pm 0.57$ \\
\hline 20 & 18/07/2007 & $=$ & 8.45 & 166 & 0.5 & 91.0 & 14.0 & 1.0 & 0.0 & 0.0 & 0.0 & 28.0 & 6.0 & 0.6 & $=73.8$ & $=10.4$ & 9.6 & $5.25 \pm 0.58$ \\
\hline 21 & 18/07/2007 & 8.7 & 8.58 & 140 & $=$ & $=$ & $=$ & $=$ & $=$ & $=$ & $=$ & $=$ & $=$ & $=$ & $=74.6$ & $=10.6$ & 10.3 & $=$ \\
\hline 22 & $17 / 07 / 2007$ & 26.0 & 7.05 & 3580 & 2.1 & 159.0 & 444.0 & 780.0 & 0.0 & 472.0 & 11.0 & 188.0 & 50.0 & 21.9 & $=66.4$ & $=9.6$ & 10.5 & $2.73 \pm 0.52$ \\
\hline 23 & $17 / 07 / 2007$ & 9.2 & 8.40 & 123 & 0.6 & 54.0 & 12.0 & 1.0 & 0.0 & 0.0 & 0.0 & 16.0 & 5.0 & 0.4 & $=74.0$ & $=10.6$ & 10.4 & $3.81 \pm 0.54$ \\
\hline 24 & $17 / 07 / 2007$ & 10.0 & 7.44 & 300 & 0.5 & 155.0 & 13.0 & 1.0 & 0.0 & 0.0 & 0.0 & 48.0 & 7.0 & 3.8 & $=62.8$ & $=9.3$ & 11.6 & $3.78 \pm 0.54$ \\
\hline 25 & $17 / 07 / 2007$ & 7.1 & 7.70 & 368 & 0.8 & 174.0 & 13.0 & 2.0 & 1.0 & 0.0 & 0.0 & 52.0 & 9.0 & 2.4 & $=63.5$ & $=9.7$ & 14.3 & $4.97 \pm 0.57$ \\
\hline 26 & $17 / 07 / 2007$ & 9.0 & 7.90 & 228 & 0.6 & 122.0 & 17.0 & 2.0 & 0.0 & 0.0 & 0.0 & 44.0 & 4.0 & 2.8 & $=64.8$ & $=9.4$ & 10.2 & $4.57 \pm 0.56$ \\
\hline 27 & $16 / 07 / 2007$ & 14.9 & 7.72 & 237 & 0.5 & 108.0 & 19.0 & 1.0 & 1.0 & 0.0 & 0.0 & 33.0 & 8.0 & 2.0 & $=72.9$ & $=10.5$ & 11.4 & $1.00 \pm 0.41$ \\
\hline 28 & $16 / 07 / 2007$ & 15.6 & 8.40 & 139 & 0.6 & 68.0 & 14.0 & 1.0 & 1.0 & 0.0 & 0.0 & 14.0 & 10.0 & 0.8 & $=69.0$ & $=9.8$ & 9.1 & $2.17 \pm 0.45$ \\
\hline 29 & 26/07/2007 & 14.1 & 8.50 & 220 & 0.6 & 88.0 & 19.0 & 1.0 & 1.0 & 0.0 & 0.0 & 31.0 & 5.0 & 1.8 & $=60.9$ & $=8.5$ & 6.8 & $4.97 \pm 0.55$ \\
\hline
\end{tabular}

Units: $\mathrm{T}$ in ${ }^{\circ} \mathrm{C}, \mathrm{EC}$ in $\mu \mathrm{S} / \mathrm{cm}$, hydrochemical ion concentration in $(\mathrm{mg} / \mathrm{L}), \mathrm{DQO}$ in $(\mathrm{mg} / \mathrm{L}), \delta^{2} \mathrm{H}, \delta^{18} \mathrm{O}$ and $\mathrm{d}$ in \%, and tritium content in UT.

a Field parameters.

${ }^{b}$ Deuterium excess $\left(\mathrm{d} \equiv={ }^{8} \delta^{18} \mathrm{O}-\delta^{2} \mathrm{H} \%\right.$ ).

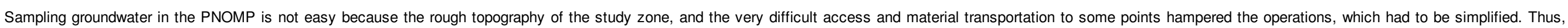

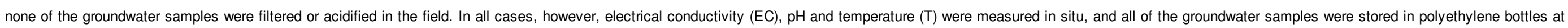
$4{ }^{\circ} \mathrm{C}$ until analysis. A total of 106 groundwater samples were obtained during the different field sampling campaigns.

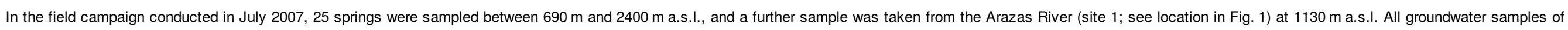

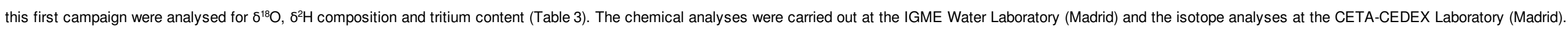

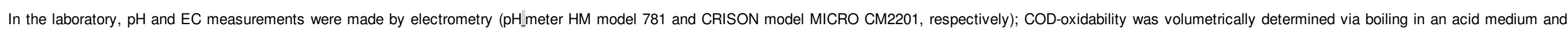

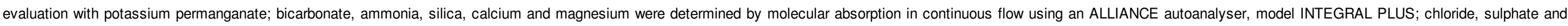

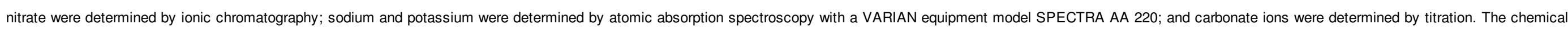
components measured in all samples from all campaigns were the same: $\mathrm{HCO}_{3}=\mathrm{Ca}^{2+}, \mathrm{Mg}^{2+}, \mathrm{Na}^{+}, \mathrm{K}^{+}, \mathrm{Cl}^{-}, \mathrm{SO}_{4}^{2-}, \mathrm{NO}_{3}^{-}, \mathrm{SiO}_{2}$.

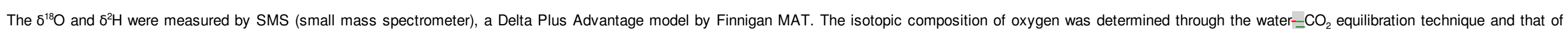

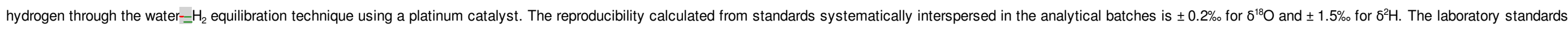

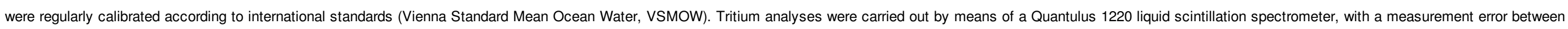
\pm 0.33 and $\pm 0.42 \mathrm{TU}$. 


\section{elsevier_STOTEN_17069}

Between May 2011 and April 2012, following a monthly schedule, groundwater samples were obtained from six springs (Table 2). In this case, the groundwater samples were only analysed for $\delta^{18} \mathrm{O}$ and $\delta^{2} \mathrm{H}($ see Table 4$)$.

Finally, in September 2013, groundwater samples were obtained from four springs (see Table 2). The isotopic analyses included $\delta^{18} \mathrm{O}, \delta^{2} \mathrm{H}, \delta^{34} \mathrm{~S}_{\mathrm{SO}_{4}}$, and $\delta^{18} \mathrm{O}_{\mathrm{SO}_{4}}\left(\mathrm{Table}^{5}\right) \cdot\left(\mathrm{see} \mathrm{Table}_{4 . \mathrm{H}}\right.$

Table 4 Representative physical, chemical and isotopic data of groundwater in the study area during the field campaigns conducted in 2011 and 2012.

\begin{tabular}{|c|c|c|c|c|c|c|c|c|c|c|c|c|c|c|c|c|c|c|c|}
\hline Sampling point code & Date & $\left.T t^{2}\right)$ & $\mathrm{pH} \mathrm{H}^{\mathrm{a}}$ & $\mathrm{EC}\left({ }^{2}{ }^{\mathrm{y}}\right.$ & $\mathrm{HCO}_{3}$ & $\mathrm{SO}_{4}$ & $\mathrm{Cl}$ & $\mathrm{NO}_{3}$ & $\mathrm{~F}$ & $\mathrm{Na}$ & K & $\mathrm{Ca}$ & $\mathrm{Mg}$ & $\mathrm{SIO}_{2}$ & $T A f^{(b)}$ & $\operatorname{TDS}\left(^{\circ}+\right.$ & $\delta^{2} \mathrm{H}$ & $\delta^{18} \mathrm{O}$ & $\left.d f^{d}\right)$ \\
\hline 19 & $24 / 05 / 2011$ & 6.2 & 8.08 & 182 & 132.0 & 0.6 & 0.9 & 0.2 & 0.0 & 1.1 & 0.0 & 45.6 & 1.4 & 132.0 & 132.0 & 140 & $=73.3$ & $=10.8$ & 12.9 \\
\hline 9 & $24 / 05 / 2011$ & 8.4 & 8.48 & 148 & 107.2 & 1.2 & 0.1 & 0.3 & 0.0 & 0.3 & 0.0 & 34.7 & 2.5 & 111.3 & 111.3 & 104 & $=76.1$ & $=10.6$ & 9.1 \\
\hline 4 & $24 / 05 / 2011$ & 8.0 & 8.05 & 298 & 165.0 & 29.5 & 1.1 & 0.6 & 0.0 & 1.1 & 0.0 & 51.3 & 15.4 & 165.0 & 165.0 & 252 & $=72.2$ & $=10.0$ & 7.5 \\
\hline 23 & $25 / 05 / 2011$ & 8.2 & 8.45 & 113 & 84.8 & 1.2 & 0.2 & 1.0 & 0.0 & 0.1 & 0.0 & 24.1 & 2.3 & 86.4 & 86.4 & 84 & $=74.2$ & $=10.8$ & 11.8 \\
\hline 29 & $26 / 05 / 2011$ & 9.2 & 8.19 & 88 & 101.7 & 2.7 & 0.1 & 0.8 & 0.0 & 0.1 & 0.0 & 31.2 & 2.2 & 101.7 & 101.7 & 96 & $=71.0$ & $=10.3$ & 11.9 \\
\hline 19 & $30 / 06 / 2011$ & 10.6 & 8.16 & 210 & 73.5 & 0.9 & 0.1 & 0.5 & 0.0 & 0.2 & 0.0 & 35.6 & 1.8 & 73.5 & 73.5 & 128 & $=69.5$ & $=10.6$ & 15.2 \\
\hline 9 & $30 / 06 / 2011$ & 13.9 & 8.89 & 140 & 52.6 & 1.3 & 0.2 & 0.4 & 0.0 & 0.4 & 0.0 & 22.8 & 2.6 & 54.5 & 54.5 & 88 & $=72.4$ & $=10.9$ & 14.4 \\
\hline 4 & $29 / 06 / 2011$ & 9.5 & 7.93 & 380 & 88.5 & 30.0 & 0.8 & 0.6 & 0.0 & 1.1 & 0.0 & 44.4 & 14.3 & 88.5 & 88.5 & 236 & $=71.3$ & $=10.3$ & 10.9 \\
\hline 23 & $29 / 06 / 2011$ & 10.3 & 8.73 & 117 & 43.6 & 1.5 & 0.3 & 0.7 & 0.0 & 0.1 & 0.0 & 16.4 & 1.4 & 43.6 & 43.6 & 80 & $=68.0$ & $=10.2$ & 13.3 \\
\hline 29 & $29 / 06 / 2011$ & 11.3 & 8.41 & 222 & 65.8 & 7.5 & 0.2 & 0.9 & 0.0 & 0.2 & 0.0 & 28.9 & 4.0 & 65.8 & 65.8 & 132 & $=65.3$ & $=9.5$ & 10.8 \\
\hline 19 & $30 / 07 / 2011$ & 12.0 & 8.42 & 180 & 59.7 & 1.3 & 0.1 & 0.4 & 0.0 & 0.3 & 0.0 & 37.4 & 1.8 & 61.4 & 61.4 & 124 & $=69.1$ & $=9.5$ & 7.2 \\
\hline 9 & $30 / 07 / 2011$ & 12.9 & 8.19 & 170 & 62.9 & 2.2 & 0.1 & 0.4 & 0.0 & 0.4 & 0.0 & 38.3 & 4.6 & 66.2 & 66.2 & 136 & $=70.6$ & $=9.6$ & 5.9 \\
\hline 4 & $30 / 07 / 2011$ & 10.7 & 7.89 & 290 & 87.7 & 37.9 & 0.7 & 0.6 & 0.0 & 1.1 & 0.0 & 52.8 & 15.6 & 87.7 & 87.7 & 260 & $=71.3$ & $=9.9$ & 7.9 \\
\hline 23 & $31 / 07 / 2011$ & 11.3 & 8.20 & 120 & 47.6 & 2.2 & 0.1 & 0.6 & 0.0 & 0.7 & 0.0 & 24.8 & 2.3 & 47.6 & 47.6 & 120 & $=72.3$ & $=9.9$ & 6.5 \\
\hline 29 & $31 / 07 / 2011$ & 11.6 & 8.41 & 230 & 64.6 & 14.0 & 0.2 & 0.9 & 0.0 & 0.5 & 0.0 & 43.4 & 7.2 & 68.8 & 68.8 & 176 & $=66.3$ & $=9.2$ & 7.1 \\
\hline 19 & $25 / 08 / 2011$ & 10.7 & 7.94 & 230 & 81.4 & 2.0 & 0.3 & 1.5 & 0.0 & 0.3 & 0.0 & 41.4 & 2.3 & 81.4 & 81.4 & 144 & $=52.2$ & $=8.3$ & 14.3 \\
\hline 9 & $24 / 08 / 2011$ & 6.5 & 8.56 & 150 & 66.7 & 4.4 & 0.2 & 1.2 & 0.0 & 0.3 & 0.0 & 26.9 & 5.0 & 66.7 & 66.7 & 108 & $=67.2$ & $=9.9$ & 12.2 \\
\hline 4 & $24 / 08 / 2011$ & 10.0 & 8.36 & 400 & 107.3 & 54.4 & 0.9 & 0.8 & 0.0 & 1.0 & 0.0 & 51.7 & 16.0 & 107.3 & 107.3 & 244 & $=70.8$ & $=10.9$ & 16.4 \\
\hline 29 & $26 / 08 / 2011$ & 11.4 & 8.28 & 180 & 89.5 & 6.3 & 0.3 & 1.1 & 0.0 & 0.3 & 0.0 & 39.3 & 3.8 & 92.4 & 92.4 & 148 & $=55.6$ & $=8.7$ & 13.8 \\
\hline 18 & $25 / 08 / 2011$ & 8.9 & 8.05 & 150 & 81.2 & 1.4 & 0.3 & 2.0 & 0.0 & 0.2 & 0.0 & 36.4 & 0.9 & 81.2 & 81.2 & 124 & $=45.3$ & $=7.4$ & 13.5 \\
\hline 19 & $30 / 09 / 2011$ & 10.8 & 8.02 & 365 & 203.1 & 1.3 & 0.3 & 0.6 & 0.0 & 0.2 & 0.0 & 43.5 & 2.4 & 203.1 & 203.1 & 188 & $=55.1$ & $=8.4$ & 11.9 \\
\hline 9 & $30 / 09 / 2011$ & 5.8 & 7.88 & 194 & 114.1 & 5.1 & 0.2 & 1.5 & 0.0 & 0.0 & 0.0 & 24.5 & 4.9 & 114.1 & 114.1 & 112 & $=66.0$ & $=10.2$ & 15.4 \\
\hline 4 & $30 / 09 / 2011$ & 8.1 & 7.83 & 356 & 142.0 & 46.1 & 0.3 & 0.7 & 0.0 & 0.1 & 0.0 & 40.2 & 12.6 & 142.0 & 142.0 & 216 & $=68.8$ & $=10.5$ & 14.9 \\
\hline 23 & 29/09/2011 & 12.6 & 8.34 & 100 & 107.4 & 3.4 & 0.2 & 1.2 & 0.0 & 0.0 & 0.0 & 24.2 & 3.1 & 113.8 & 113.8 & 104 & $=54.8$ & $=8.5$ & 13.5 \\
\hline 29 & 29/09/2011 & 10.9 & 8.28 & 100 & 132.3 & 12.7 & 0.2 & 1.0 & 0.0 & 0.1 & 0.0 & 29.4 & 5.8 & 136.4 & 136.4 & $=$ & $=59.2$ & $=9.1$ & 13.3 \\
\hline 18 & $30 / 09 / 2011$ & 10.3 & 8.05 & 273 & 156.0 & 2.5 & 1.4 & 4.1 & 0.0 & 0.9 & 2.0 & 44.1 & 2.6 & 156.0 & 156.0 & 224 & $=52.3$ & $=8.3$ & 13.8 \\
\hline 19 & $26 / 10 / 2011$ & 8.7 & 8.34 & 147 & 146.2 & 1.5 & 0.5 & 1.0 & 0.0 & 0.2 & 0.0 & 45.4 & 1.9 & 146.2 & 146.2 & 116 & $=61.2$ & $=9.6$ & 15.5 \\
\hline 9 & $26 / 10 / 2011$ & 4,9 & 5.60 & 210 & 124.3 & 3.3 & 0.5 & 1.1 & 0.0 & 0.3 & 0.0 & 33.2 & 3.9 & 124.3 & 124.3 & 116 & $=68.6$ & $=10.4$ & 14.6 \\
\hline
\end{tabular}




\section{elsevier_STOTEN_17069}

\begin{tabular}{|c|c|c|c|c|c|c|c|c|c|c|c|c|c|c|c|c|c|c|c|}
\hline 4 & $26 / 10 / 2011$ & 6.9 & 5.20 & 390 & 139.8 & 54.6 & 0.3 & 0.7 & 0.0 & 0.5 & 0.0 & 50.1 & 14.8 & 139.8 & 139.8 & 248 & $=68.5$ & $=10.3$ & 13.7 \\
\hline 23 & 29/10/2011 & 7.9 & $=$ & 187 & 110.9 & 1.5 & 0.3 & 1.0 & 0.0 & 0.2 & 0.0 & 28.0 & 2.0 & 114.2 & 114.2 & 72 & $=68.6$ & $=10.7$ & 17.4 \\
\hline 29 & $29 / 10 / 2011$ & 9.5 & $=$ & 209 & 125.4 & 2.4 & 0.3 & 1.0 & 0.0 & 0.2 & 0.0 & 35.9 & 2.9 & 125.4 & 125.4 & 96 & $=65.7$ & $=10.4$ & 17.3 \\
\hline 18 & $26 / 10 / 2011$ & 8.3 & 5.20 & 160 & 125.0 & 1.2 & 1.1 & 1.4 & 0.0 & 0.7 & 0.5 & 39.4 & 0.8 & 125.0 & 125.0 & 116 & $=61.0$ & $=9.2$ & 12.7 \\
\hline 19 & 29/11/2011 & 6.7 & $=$ & 160 & 149.4 & 0.6 & 0.2 & 0.2 & 0.0 & 0.0 & 0.0 & 37.1 & 1.4 & 153.0 & 153.0 & 164 & $=74.1$ & $=11.1$ & 14.5 \\
\hline 9 & 29/11/2011 & 5.4 & $=$ & 140 & 115.6 & 2.6 & 0.2 & 1.0 & 0.0 & 0.0 & 0.0 & 26.5 & 3.8 & 115.6 & 115.6 & 108 & $=76.1$ & $=11.5$ & 15.7 \\
\hline 4 & $29 / 11 / 2011$ & 7.4 & $=$ & 270 & 137.9 & 31.5 & 0.4 & 0.9 & 0.1 & 0.4 & 0.0 & 36.3 & 10.3 & 137.9 & 137.9 & 200 & $=68.1$ & $=10.2$ & 13.3 \\
\hline 23 & $30 / 11 / 2011$ & 3.7 & $=$ & 176 & 115.8 & 2.4 & 0.2 & 0.8 & 0.0 & 0.0 & 0.0 & 25.5 & 2.4 & 118.2 & 118.2 & 116 & $=68.6$ & $=10.5$ & 15.7 \\
\hline 29 & $30 / 11 / 2011$ & 7.4 & $=$ & 220 & 126.7 & 8.0 & 0.2 & 0.9 & 0.0 & 0.0 & 0.0 & 30.0 & 4.5 & 131.1 & 131.1 & 144 & $=67.3$ & $=10.2$ & 14.2 \\
\hline 18 & 29/11/2011 & 9.0 & $=$ & 120 & 118.0 & 1.0 & 0.4 & 3.5 & 0.0 & 0.4 & 0.0 & 31.6 & 1.1 & 121.3 & 121.3 & 124 & $=81.5$ & $=12.1$ & 15.1 \\
\hline 9 & $23 / 12 / 2011$ & 5.6 & 8.30 & 170 & 122.0 & 4.5 & 0.1 & 1.0 & 0.0 & 0.0 & 0.0 & 30.8 & 5.6 & 122.0 & 122.0 & 108 & $=79.1$ & $=11.6$ & 13.6 \\
\hline 4 & 23/12/2011 & 8.5 & 7.85 & 370 & 144.7 & 40.4 & 0.3 & 0.7 & 0.0 & 0.0 & 0.0 & 46.7 & 12.1 & 144.7 & 144.7 & 200 & $=69.8$ & $=10.6$ & 15.2 \\
\hline 9 & 28/02/2012 & 8.4 & 6.40 & 190 & 112.6 & 5.0 & 0.2 & 1.2 & 0.0 & 0.1 & 0.0 & 29.1 & 5.3 & 112.6 & 112.6 & 112 & $=74.1$ & $=11.5$ & 17.5 \\
\hline 19 & 28/03/2012 & 8.4 & 6.40 & 200 & 133.6 & 1.1 & 0.2 & 0.5 & 0.0 & 0.0 & 0.0 & 41.4 & 1.1 & 133.6 & 133.6 & 136 & $=73.8$ & $=11.5$ & 18.0 \\
\hline 9 & 28/03/2012 & 8.5 & 6.40 & 160 & 108.0 & 2.2 & 0.2 & 1.1 & 0.0 & 0.0 & 0.0 & 33.0 & 3.5 & 108.0 & 108.0 & 108 & $=78.2$ & $=11.7$ & 15.0 \\
\hline 4 & 28/03/2012 & 8.1 & 6.40 & 350 & 126.2 & 53.6 & 0.4 & 0.8 & 0.0 & 0.0 & 0.0 & 42.4 & 12.5 & 126.2 & 126.2 & 244 & $=70.5$ & $=10.7$ & 14.8 \\
\hline 23 & $27 / 03 / 2012$ & 8.5 & 6.40 & 140 & 101.6 & 2.0 & 0.3 & 1.5 & 0.0 & 0.0 & 0.0 & 28.1 & 2.0 & 101.6 & 101.6 & 104 & $=69.2$ & $=10.7$ & 16.7 \\
\hline 29 & 28/03/2012 & $=$ & $=$ & $=$ & 101.4 & 4.8 & 0.3 & 1.5 & 0.0 & 0.0 & 0.0 & 30.9 & 2.9 & 101.4 & 101.4 & 120 & $=64.5$ & $=9.9$ & 15.0 \\
\hline 18 & 28/03/2012 & 8.6 & 6.40 & 110 & 94.3 & 1.2 & 0.4 & 2.0 & 0.0 & 0.3 & 0.0 & 31.7 & 0.7 & 94.3 & 94.3 & 108 & $=73.8$ & $=10.9$ & 13.0 \\
\hline 9 & $24 / 04 / 2012$ & $=$ & $=$ & $=$ & 114.7 & 3.1 & 0.2 & 1.1 & 0.0 & 0.9 & 0.0 & 33.4 & 4.4 & 115.8 & 115.8 & 108 & $=74.6$ & $=11.0$ & 13.6 \\
\hline 4 & $24 / 04 / 2012$ & $=$ & $=$ & $=$ & 124.0 & 36.8 & 0.3 & 0.8 & 0.0 & 0.3 & 0.0 & 47.5 & 13.6 & 124.0 & 124.0 & 204 & $=68.7$ & $=10.1$ & 12.4 \\
\hline 29 & $24 / 04 / 2012$ & $=$ & $=$ & $=$ & 125.3 & 4.9 & 0.2 & 0.7 & 0.0 & 0.4 & 0.0 & 38.8 & 4.6 & 131.6 & 131.6 & 128 & $=64.3$ & $=9.6$ & 12.6 \\
\hline
\end{tabular}

Units: $\mathrm{T}$ in ${ }^{\circ} \mathrm{C}$, EC in $\mu \mathrm{S} / \mathrm{cm}$, hydrochemical ion concentration in (mg/L), TA (mg/L), TDS in \%, and isotope content in \%o,

${ }^{\text {a }}$ Field parameters.

b Total alkalinity.

c Total dissolved solids.

d Deuterium excess $\left(\mathrm{d} \equiv=-8 \delta^{18} \mathrm{O}-\delta^{2} \mathrm{H} \% \mathrm{o}\right)$.

Table 5 Representative physical, chemical and isotopic data for groundwater in the study area for the field campaign conducted in 2013.

\begin{tabular}{|c|c|c|c|c|c|c|c|c|c|c|c|c|c|c|c|c|c|c|}
\hline Sampling point code & Date & $T\left(t^{2}\right)$ & $\mathrm{pH} \mathrm{H}^{\mathrm{a}}$ & $E C(-a)$ & $\mathrm{HCO}_{3}$ & $\mathrm{SO}_{4}$ & $\mathrm{Cl}$ & $\mathrm{NO}_{3}$ & $\mathrm{Na}$ & $\mathrm{K}$ & $\mathrm{Ca}$ & $\mathrm{Mg}$ & $\mathrm{SIO}_{2}$ & $\delta^{2} \mathrm{H}$ & $\delta^{18} \mathrm{O}$ & $d\left(p^{b}\right)$ & $\mathrm{\delta}^{18} \mathrm{O}_{\mathrm{SO}_{4}}$ & $\delta^{34} \mathrm{~S}_{\mathrm{SO}_{4}}$ \\
\hline $6 \mathrm{~A}$ & $15 / 09 / 2013$ & 9.2 & 7.2 & 2190 & 114.0 & 1340.8 & 2.5 & 0.1 & 7.3 & 0.7 & 424.0 & 89.0 & 11.7 & $=74.9$ & $=11.8$ & $=13.8$ & 17.3 & 18.3 \\
\hline $6 \mathrm{~B}$ & $15 / 09 / 2013$ & 9.7 & 7.5 & 707 & 139.0 & 245.8 & 0.8 & 0.4 & 1.9 & 0.2 & 102.0 & 32.0 & 4.1 & $=72.4$ & $=10.7$ & $=13.2$ & 16.2 & 16.9 \\
\hline 10 & $15 / 09 / 2013$ & 7.9 & 7.8 & 350 & 99.0 & 85.6 & 0.4 & 0.2 & 1.9 & 0.2 & 39.0 & 18.0 & 4.5 & $=75.0$ & $=11.7$ & $=13.5$ & 14.0 & 13.7 \\
\hline
\end{tabular}




\section{elsevier_STOTEN_17069}

\begin{tabular}{|c|c|c|c|c|c|c|c|c|c|c|c|c|c|c|c|c|c|c|}
\hline 22 & $15 / 09 / 2013$ & 24.8 & 7.2 & 3150 & 209.0 & 491.5 & 639.1 & 0.2 & 510.0 & 16.3 & 166.0 & 32.0 & 17.8 & $=64.3$ & $-=9.6$ & $=12.6$ & 15.0 & 17.7 \\
\hline Rainfallc & $30 / 09 / 2013$ & $=$ & $=$ & $=$ & 5.0 & 1.7 & 0.31 & 1.14 & 0.24 & 0.1 & 1.0 & 1.0 & 0.0 & $=34.8$ & $=6.6$ & 18.0 & $=$ & $=$ \\
\hline
\end{tabular}

Units: $\mathrm{T}$ in ${ }^{\circ} \mathrm{C}$, EC in $\mu \mathrm{S} / \mathrm{cm}$, hydrochemical ion concentration in $(\mathrm{mg} / \mathrm{L})$ and isotope content in \%o.

${ }^{\text {a }}$ Field parameters.

${ }^{\mathrm{b}}$ Deuterium excess $\left(\mathrm{d} \equiv=8 \delta^{18} \mathrm{O}-\delta^{2} H \% \mathrm{H}\right)$.

c Monthly accumulated rainfall at the Góriz Meteorological station.

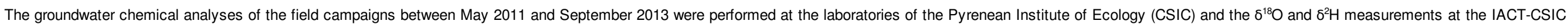

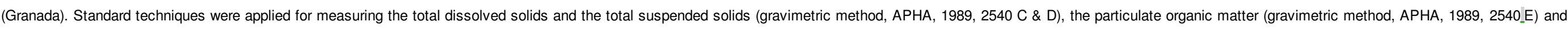

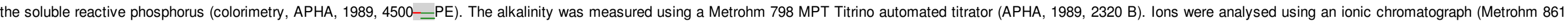

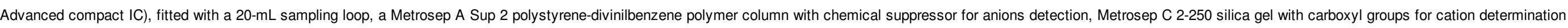

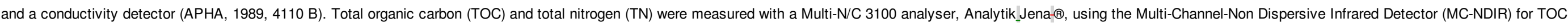

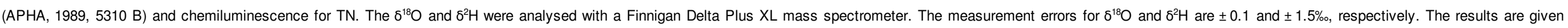
relative to the $\mathrm{V}$-SMOW standard.

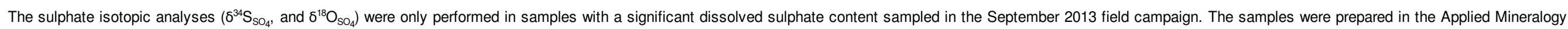

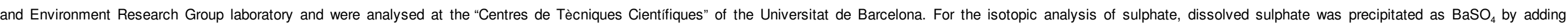

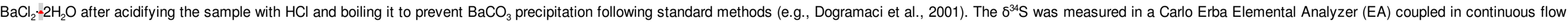

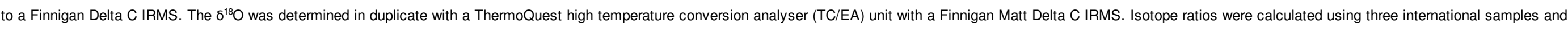

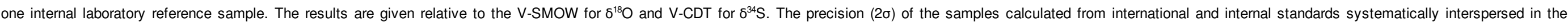
analytical batches was $\pm 0.2 \%$ for $8^{34} \mathrm{~S}$ and $\pm 0.5 \%$ for $\delta^{18} \mathrm{O}$.

\section{Results}

\subsection{Hydrogeochemistry}

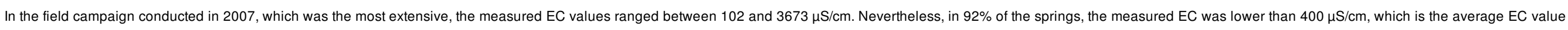

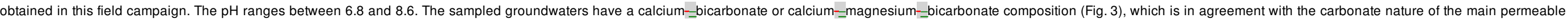

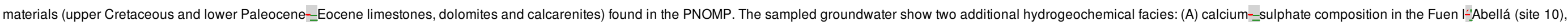

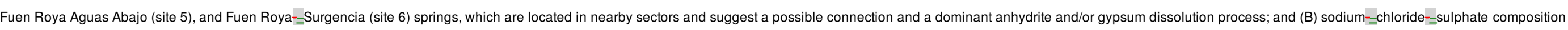

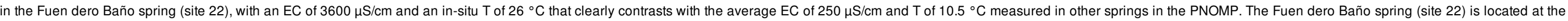
southernmost end of the PNOMP, relatively far from the other sampled springs, at the lowest altitude sector (Fig. 1). Therefore, its location could account for these differences with respect to the other sampling points located northward. 

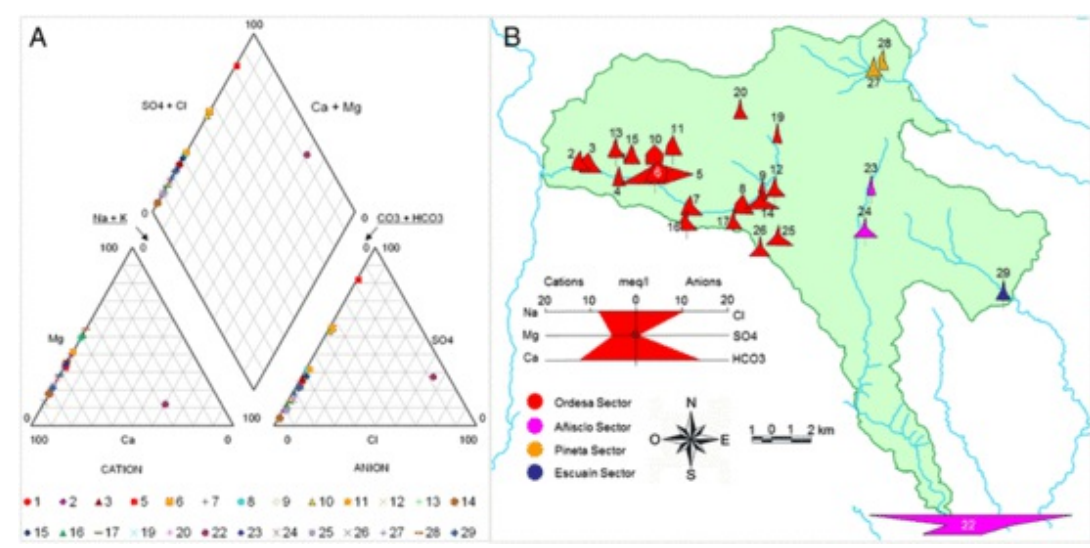

$\cdot 15+16-17+10 \cdot 20 \cdot 22 \cdot 25 \times 24 \cdot 25 \times 20+27-28 \cdot 02$

Fig. 3 Piper (A) and modified Stiff diagrams (B) for the July/2007 field campaign. The site numbers correspond to those of Table 1.

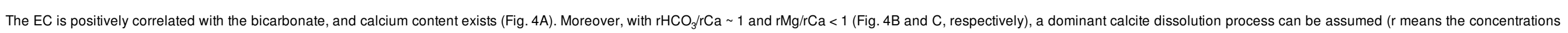
are expressed in meq/L).
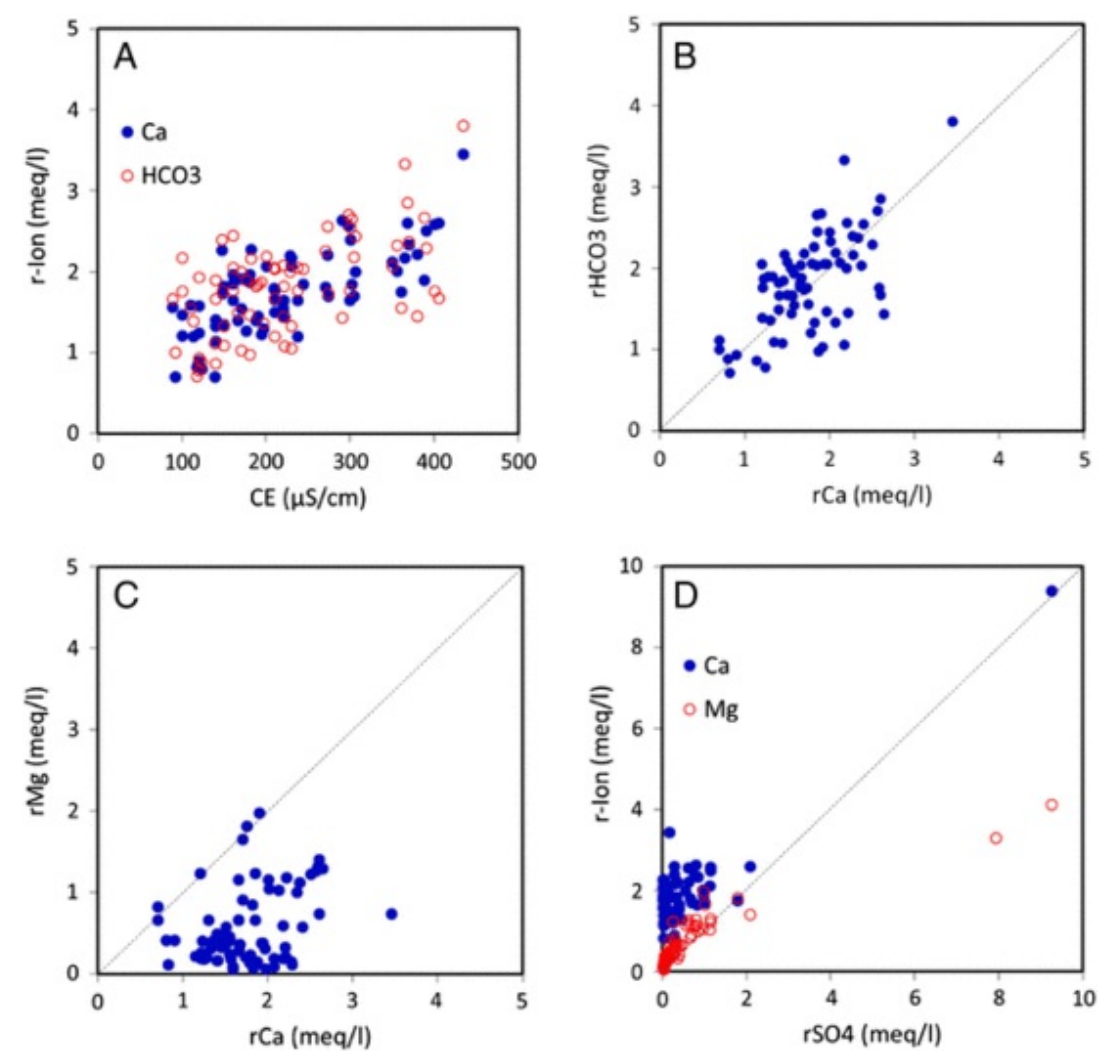

Fig. 4 Relationships between (A) EC and both $\mathrm{rHCO}_{3}$ and rCa; (B) rCa and $\mathrm{rHCO}_{3}$; (C) rCa and rMg; (D) rSO and both rMg and rCa. Field campaigns 2007 and $2011=2012$. 


\section{elsevier_STOTEN_17069}

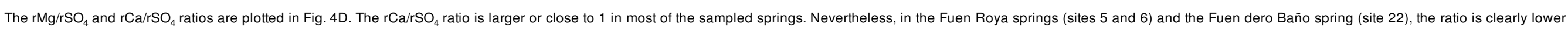

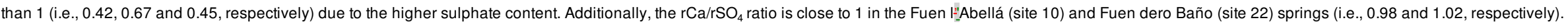

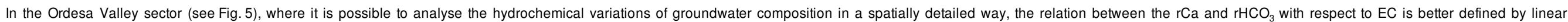
relationships (Fig. 5). Additionally, in this sector, the $\mathrm{rMg} / \mathrm{rCa}$ and $\mathrm{rCa} / \mathrm{rSO}_{4}$ ratios are directly and inversely correlated with the magnesium contents, respectively (Fig. 6).
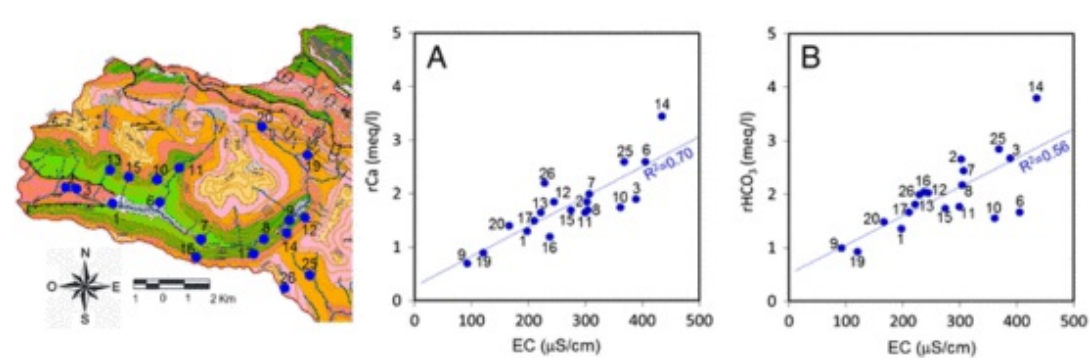

Fig. 5 Relationships between (A) $\mathrm{EC}$ and $\mathrm{rCa}$ and (B) $\mathrm{EC}$ and $\mathrm{rHCO}_{3}$ obtained from the Ordesa Valley sector during the 2007 field campaign. The numbers correspond to those of Table 1.
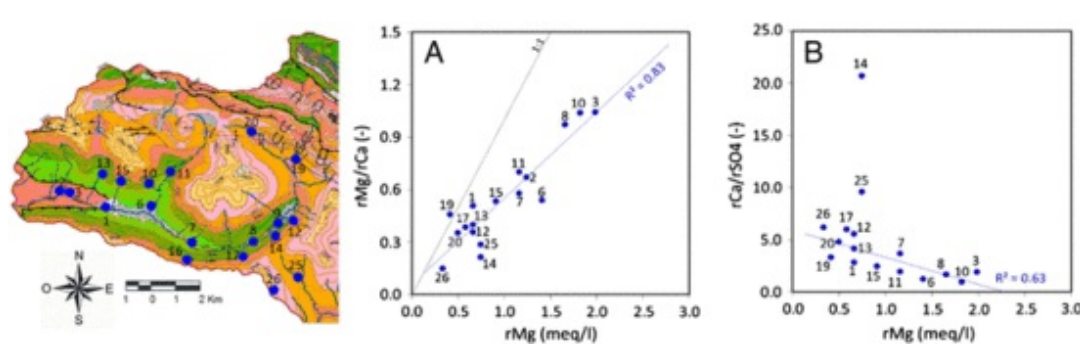

Fig. 6 Relationships between (A) $\mathrm{rMg}$ and $\mathrm{rMg} / \mathrm{rCa}$ and (B) $\mathrm{rMg}$ and $\mathrm{rCa} / \mathrm{rSO}_{4}$ obtained from the Ordesa Valley sector during the 2007 field campaign. The numbers correspond to those of Table 1.

\subsection{Stable isotopes}

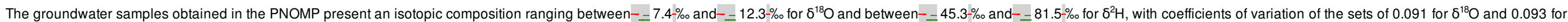
$\delta^{2} \mathrm{H}$.

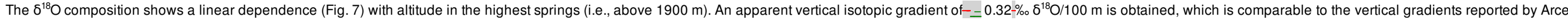
et al. (2001) and lribar and Antigüedad (1996) in other Pyrenean sectors westward. 


\section{elsevier_STOTEN_17069}

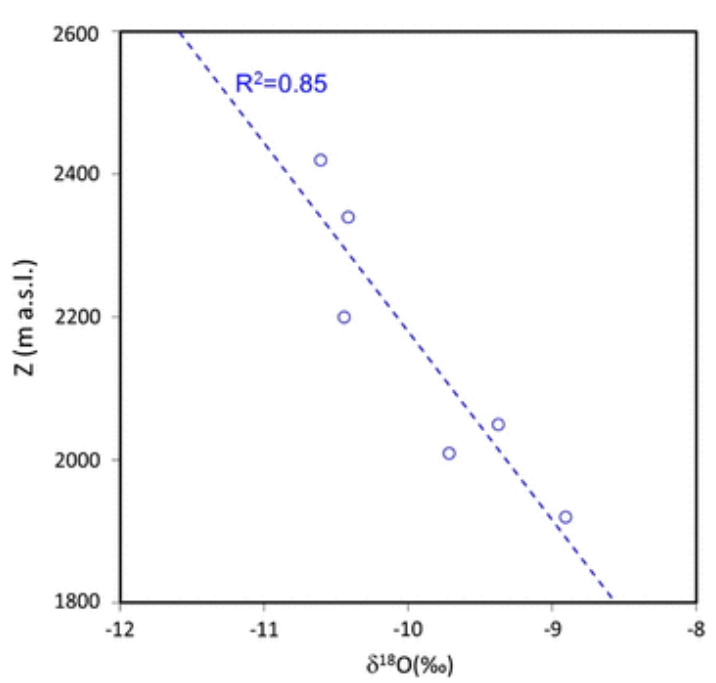

Fig. 7 Relationship between $\delta^{18} \mathrm{O}$ and altitude for samples taken in the springs located above $1900 \mathrm{~m}$ during the 2007 field campaign. The apparent altitudinal isotopic gradient is- $=0.32 \% \% \delta^{18} \mathrm{O} / 100 \mathrm{~m}$.

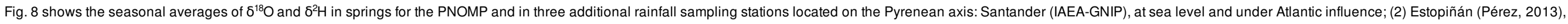

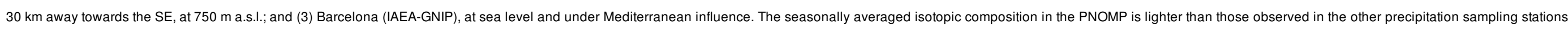

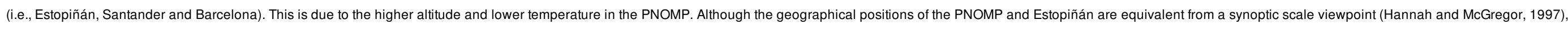

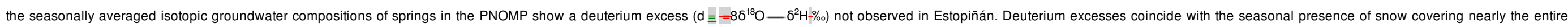
PNOMP between October and June.

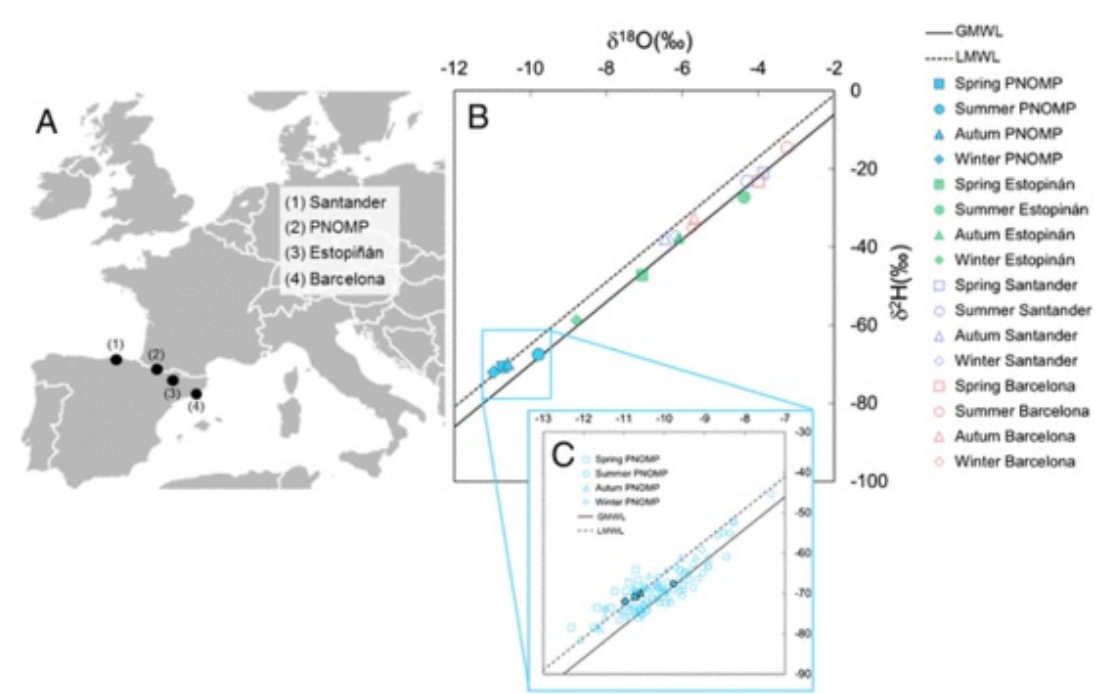

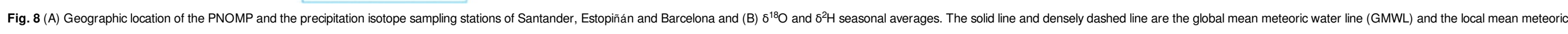
water line (LMWL) of slope $\mathrm{m} \equiv-8$ and deuterium excess of +10 and $+15 \%$, respectively. (C) Measured and seasonally averaged values of $\delta^{18} \mathrm{O}$ and $\delta^{2} \mathrm{H}$ (open and solid symbols, respectively) in the PNOMPI 


\section{elsevier_STOTEN_17069}

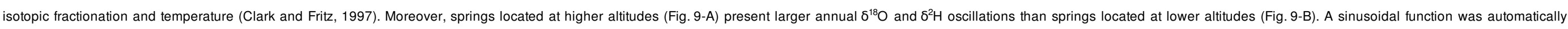

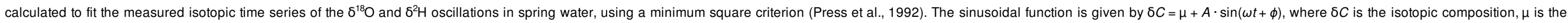
average isotopic composition, $t$ is time, $A$ is the amplitude, $\phi$ is the angular shift and $\omega$ is the angular frequency. The amplitude $A$ and the angular shift $\phi$ are parameters to be estimated.
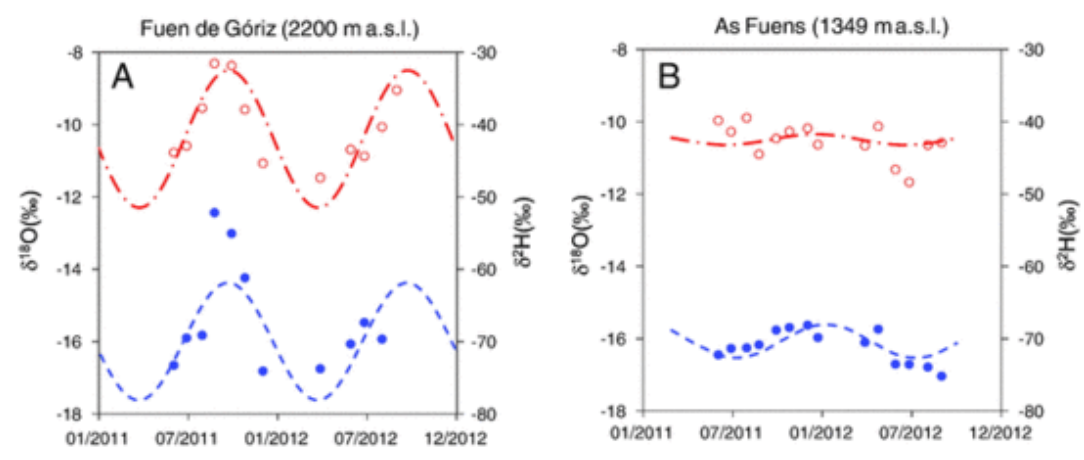

$0 \delta^{2} \mathrm{H} \quad-\cdots-1-\delta^{2} \mathrm{H}$ Sinusoidal Regression Line

$0 \delta^{10} \mathrm{O} \quad-\cdots \cdot-\delta^{180} \mathrm{O}$ Sinusoidal Regression Line

Fig. 9 Temporal evolution of the $\delta^{18} \mathrm{O}, \delta^{2} \mathrm{H}$ concentrations during the 2011-2012 field campaign at Fuen de Góriz (A) and As Fuens (B) springs.

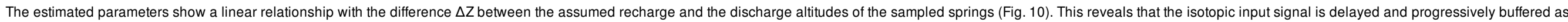
$\Delta Z$ increases.

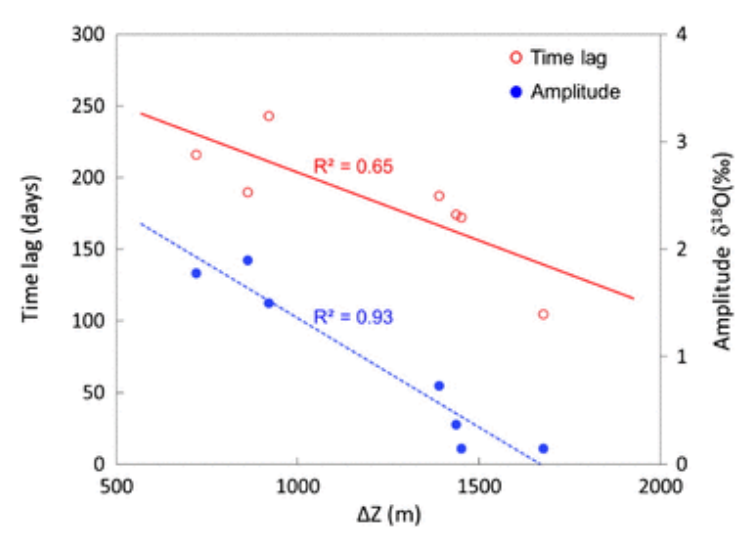

Fig. 10 Time lag and $\delta^{18} \mathrm{O}$ amplitude versus altitude difference $\Delta Z$ between recharge area and discharge site of the 2011=2012 field campaign. This time lag depends on the signal time lag and depth, so it decreases with increasing depth (Jódar et al., 2014).

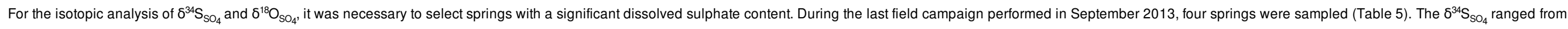
+13.7 to $+18.3 \%$ and the $\delta^{18} \mathrm{O}_{\mathrm{SO}_{4}}$ from +14 to $+17.3 \%$. The sample from the L" $\mathrm{Abella}$ spring (site 10) presents lighter isotope compositions than those obtained in the other three sampled springs (sites $6 \mathrm{~A}, 6 \mathrm{~B}$ and 22$)$ (Fig. 11$)$. 


\section{elsevier_STOTEN_17069}

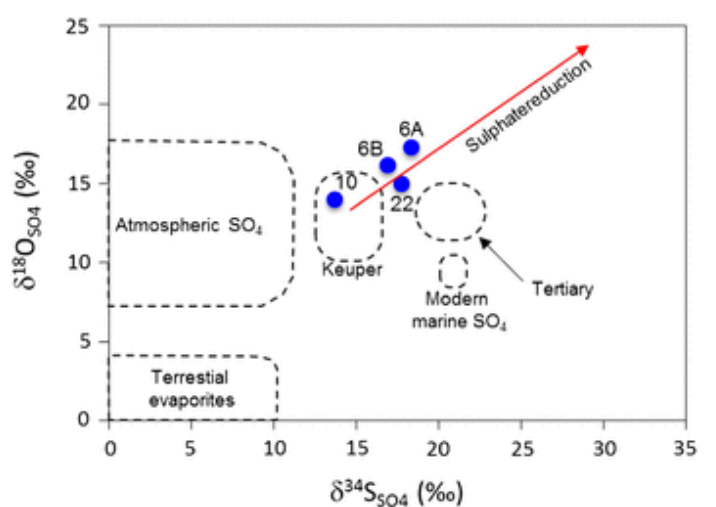

Fig. $11{ }^{18} \mathrm{O}_{\mathrm{SO}_{4}}$ vs $\delta^{34} \mathrm{~S}_{\mathrm{SO}_{4}}$ for the analysed groundwater samples (modified from Clark and Fritz, 1997). The numbers correspond to those of Table 1 .

\subsection{Tritium}

The tritium content in the groundwater was analysed only in water samples collected during the July/2007 field campaign. It ranges between $6.1 \pm 0.6 \mathrm{TU}$ and $4.6 \pm 0.6 \mathrm{TU}$, with a coefficient of variation of the set of 0.093 .

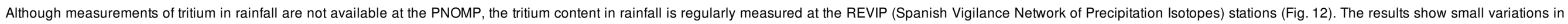
tritium concentrations during the last 15 years, with no trend (Díaz-Teijeiro et al., 2009).
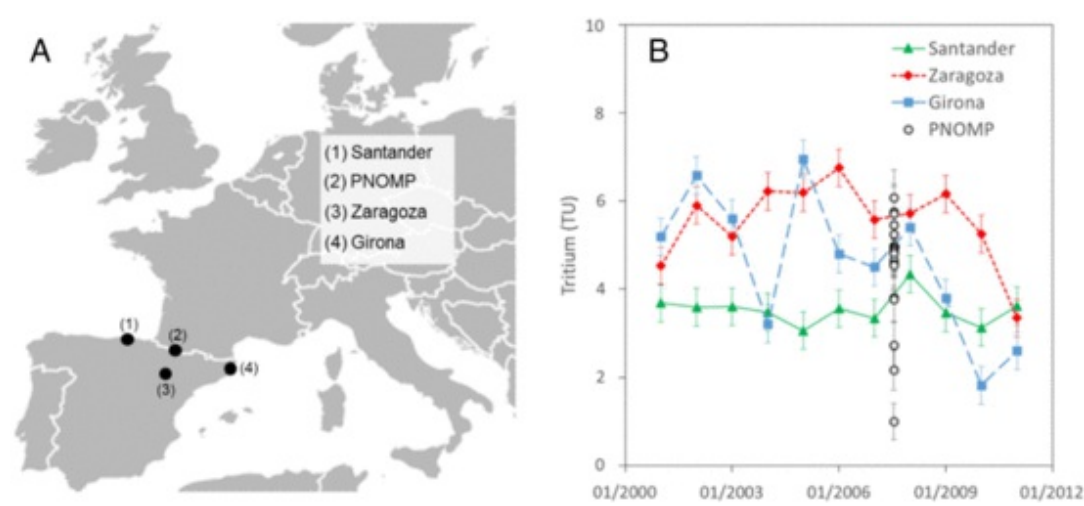

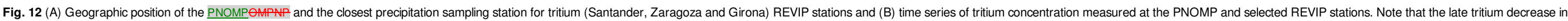
the Mediterranean stations is not produced at Santander station, which is in the path of incoming rainfall to the PNOMP.

The tritium concentration in rainfall from the stations closest to the study area is close to those measured in the sampled springs, indicating short transit times of recharge water through the aquifer.

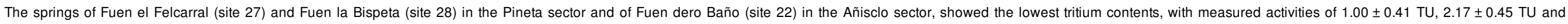
$2.73 \pm 0.52 \mathrm{TU}$, respectively

\section{Discussion}

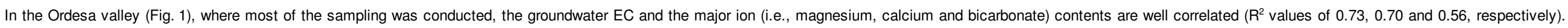

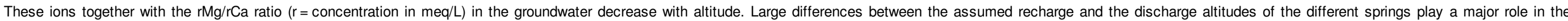

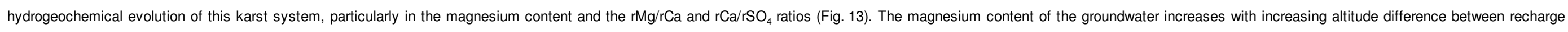
and discharge zones (Fig. 13-A and -B), while the calcium content decreases as the altitude difference increases (Fig. 13-B and -C). 


\section{elsevier_STOTEN_17069}
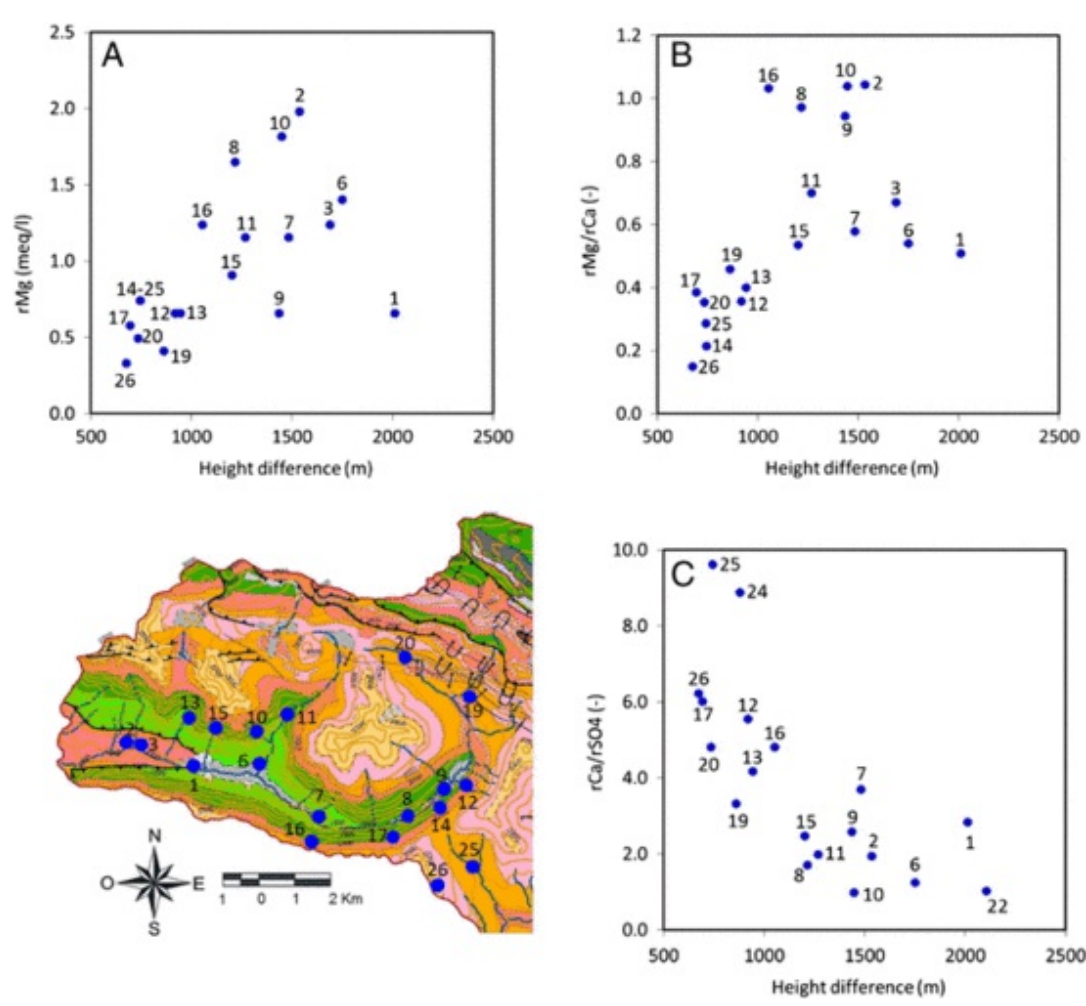

Fig. 13 Variation of the relationships $\mathrm{rMg}, \mathrm{rMg} / \mathrm{rCa}$ and $\mathrm{rCa} / \mathrm{rSO}_{4}$ regarding the altitude difference in the geographical environment of the Ordesa Valley during the 2007 field campaign. The numbers correspond to those of Table 1 .

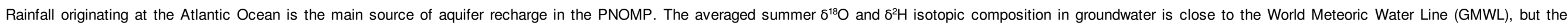

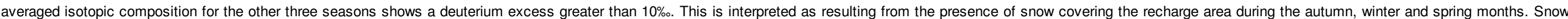

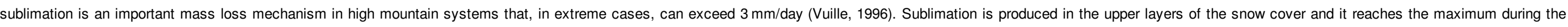

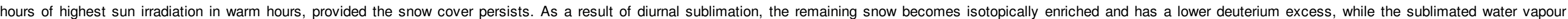

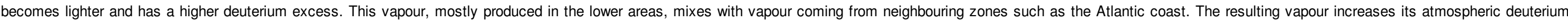

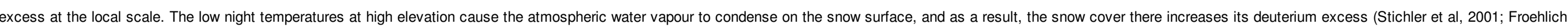

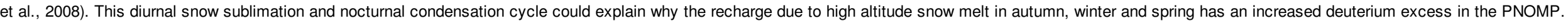

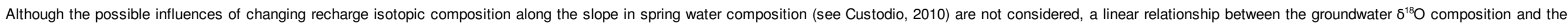

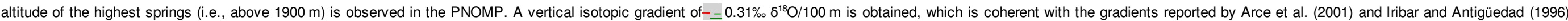

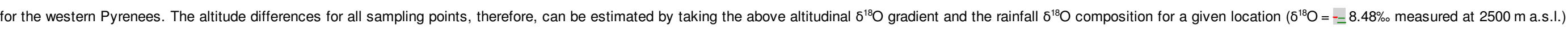
(Table 2).

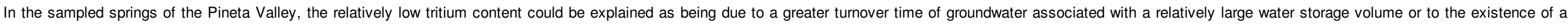

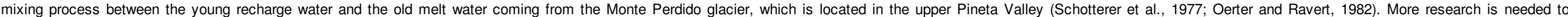

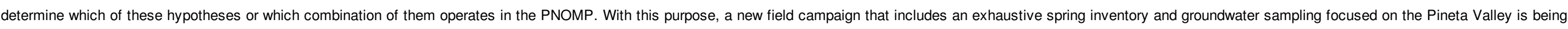
conducted. 


\section{elsevier_STOTEN_17069}

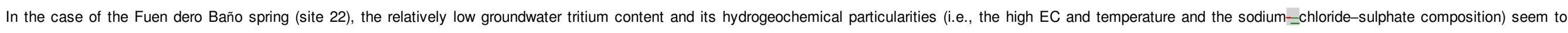

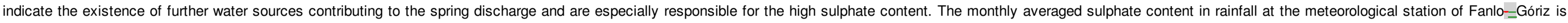

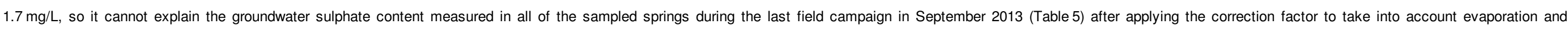
transpiration (Choi et al., 2011).

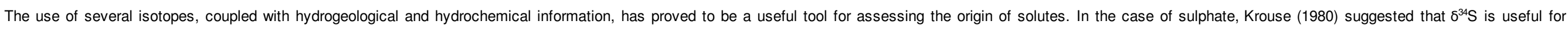

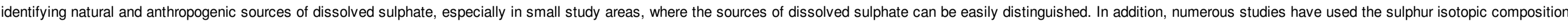
coupled with the oxygen isotopic composition of the dissolved sulphate molecule to characterize the sources of dissolved sulphate in surface and groundwater (e.g., Rock and Mayer, 2009; Tichomirowa et al., 2010).

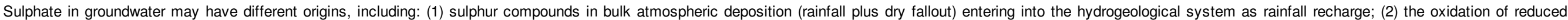

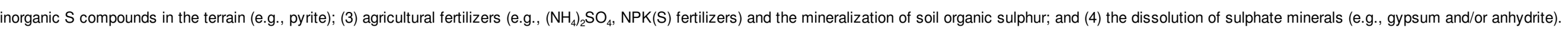

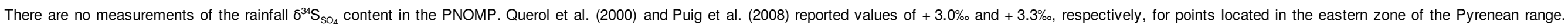

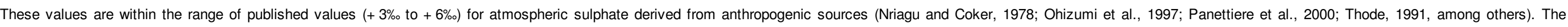

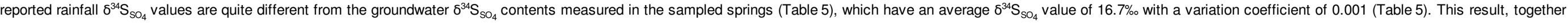

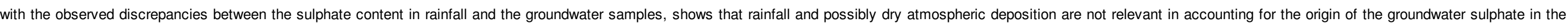
PNOMP.

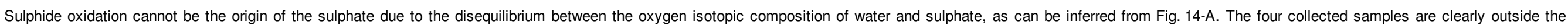

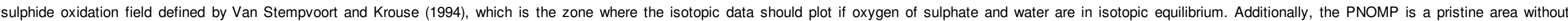
agricultural activities, which implies that neither agricultural fertilizers nor soil organic sulphur is responsible for the dissolved sulphate in the groundwater samples.
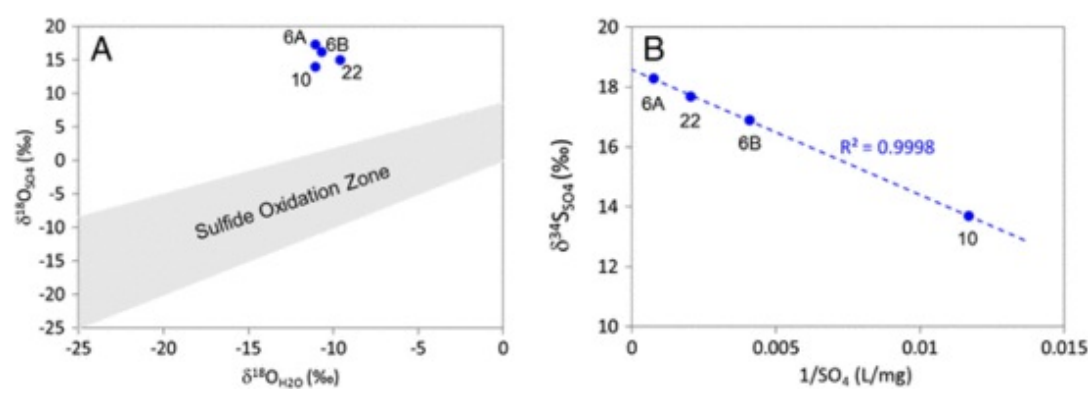

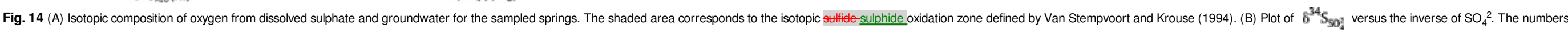
correspond to those of Table 1.

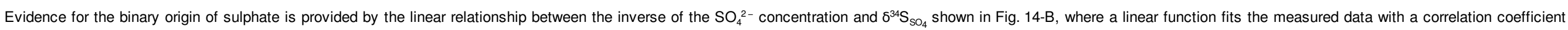

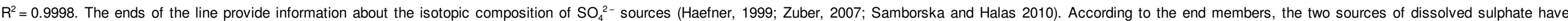

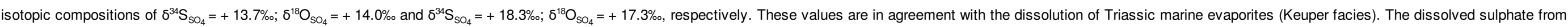

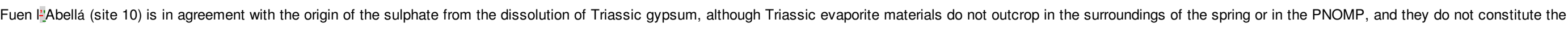

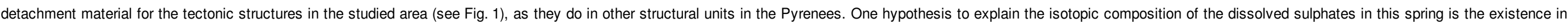

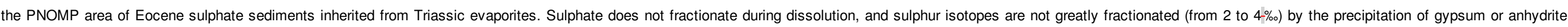

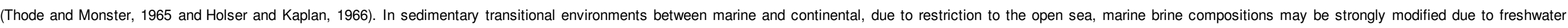

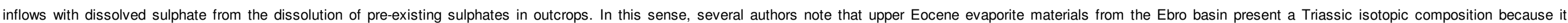

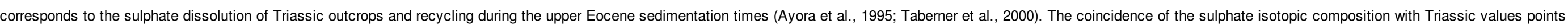




\section{elsevier_STOTEN_17069}

to this hypothesis as the most likely, given the geological setting. Nevertheless, more research is needed to determine the presence of recycled evaporites in the Tertiary materials of the PNOMP.

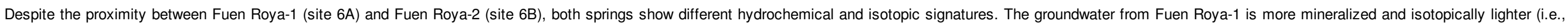

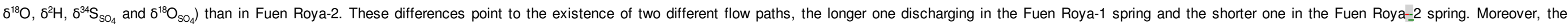

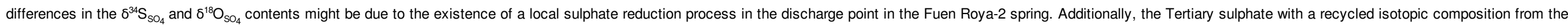
Triassic might also explain the sulphate isotope value observed in Fuen dero Baño spring (site 22) (Fig. 11).

\section{Conclusions}

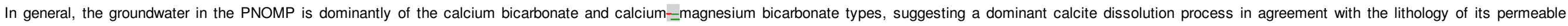

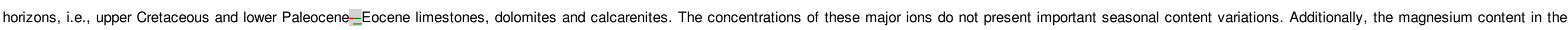

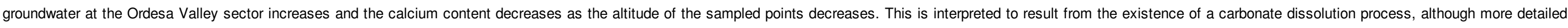

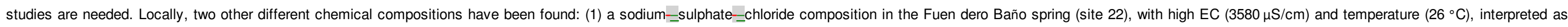
the mixing of local rainfall recharge and local highly mineralized groundwater flow; and (2) a calcium_-_sulphate composition in the Fuen Roya (sites 5, 6, 6A and 6B) and Fuen l'Abellá (site 10) springs.

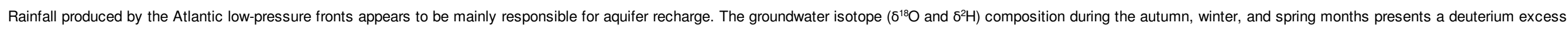

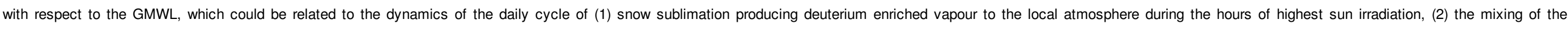

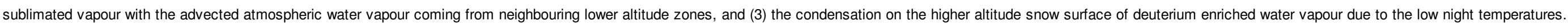
This is a preliminary result to be confirmed with further sampling and consideration of wind conditions, if this can be done.

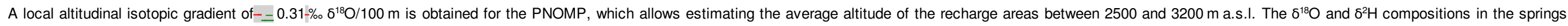

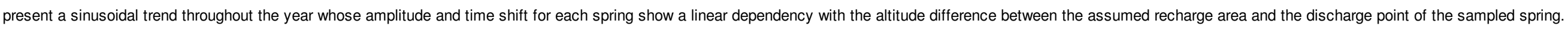

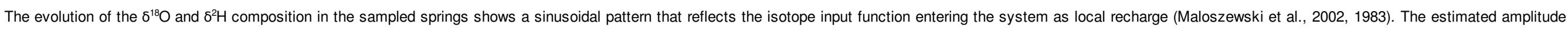

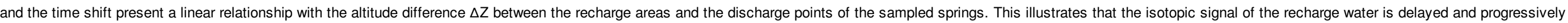
buffered as the altitude difference of the sampling point increases.

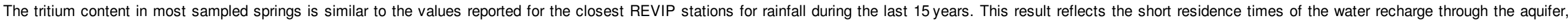

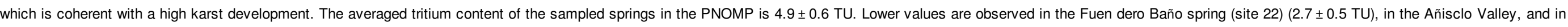

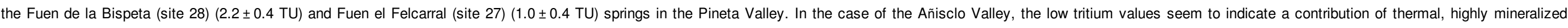

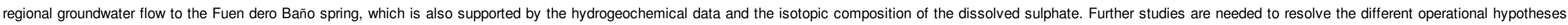
regarding the low tritium content in the sampled springs of the Pineta Valley.

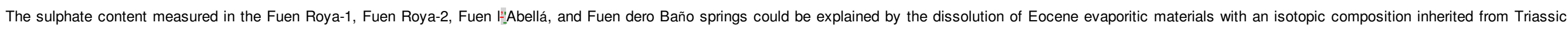

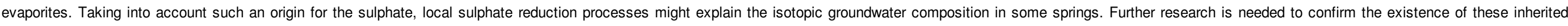
sulphates in the Eocene sedimentary materials of the PNOMP.

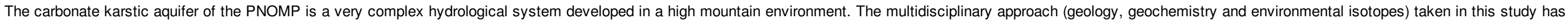
contributed to the progressive development of a hydrogeological conceptual model of aquifer system functioning

\section{Uncited references}

Alealá, 2006

Gantarelli,2011

Glaypoltat., 1980 
Gotal., 1996

Sehellart, 2002

\section{Acknowledgements}

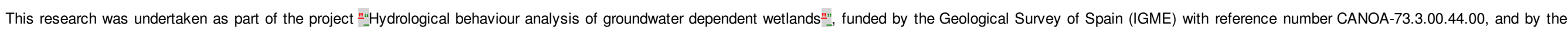

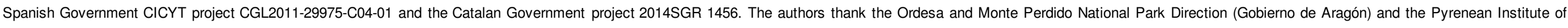

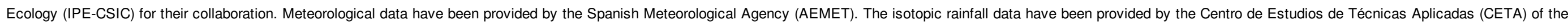
Centro de Estudios y Experimentación de Obras Públicas (CEDEX), which, together with the AEMET, manages the Spanish Monitoring Network for Isotopes in Precipitation (REVIP).

We would also like to thank the anonymous reviewers for their constructive comments and suggestions which led to a substantial improvement of the paper.

\section{References}

AEMET/IM, Atlas Climático Ibérico-_Iberian Climate Atlas, 2011, AEMET \& IM; Madrid, 1-80.

Alcalá, F.J., (2006). Recarga a los acuíferos españoles mediante balance hidrogeoquímico. Doctoral dissertation, Technical University of Catalonia (UPC). Barcelona.

APHA, Standard methods for examination of water and wastewater, In: 17 Ed-American Public Health Association, American Water Works Association, Water Pollution Control Federation, 1989.

Araguás-Araguás L., Froehlich K. and Rozanski K., Deuterium and oxygen-18 isotope composition of precipitation and atmospheric moisture, Hydrol Process 14, $2000,1341-1355$.

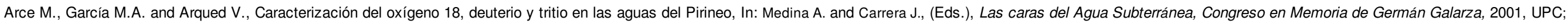
Barcelona, 387-393.

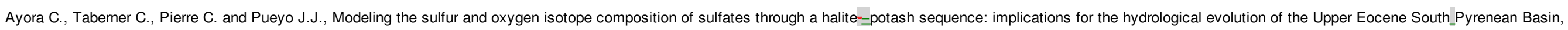
Geochem Cosmochim Acta 59, 1995, 1799-1808.

Benito Alonso J.L., Vegetación del Parque Nacional de Ordesa y Monte Perdido, 50, 2006, Publ. Consejo Protección Naturaleza Aragón; Zaragoza.

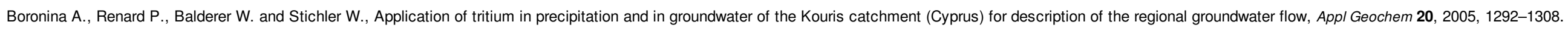

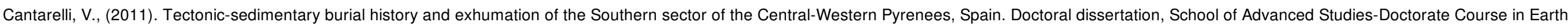
Sciences (XXIII cycle).

Carreras J. and Santanach P., El hercínico de los Pirineos, In: Geología de España, Libro Jubilar Rios, JM, 1, 1983, Instituto Geológico y Minero de España, 536-549.

CHE, Catalogación de los acuíferos de la Cuenca del Ebro, In: Oficina de Planificación Hidrológica, 1998, Confederación Hidrográfica del Ebro; Zaragoza.

Choi B.Y., Yun S.T., Mayer B. and Kim K.H., Sources and biogeochemical behavior of nitrate and sulfate in an alluvial aquifer: hydrochemical and stable isotope approaches, Appl Geochem 26 (7), 2011, 1249-1260.

Clark I.D. and Fritz P., Environmental isotopes in hydrogeology, 1997, CRC Press.

Claypool G.E., Holser W.T., Kaplan I.R., Sakai H. and Zak I., The age curves of sulfur and oxygen isotopes in marine sulfate and their mutual interpretation, Chem Geol 28, 1980, 199-260.

Collins D.N. and Gordon J.Y., Meltwater hydrology and hydrochemistry in snow- and ice-covered mountain catchments, Nord. Hydrol. 12, $1981,319-334$.

Coney P.J., Muñoz J.A., McClay K.R. and Evenchick C.A., Syntectonic burial and post-tectonic exhumation of the southern Pyrenees foreland fold-thrust belt, J Geol Soc 153 (1), $1996,9-16$.

Cortesi N., Trigo R.M., Gonzalez-Hidalgo J.C. and Ramos A.M., Modelling monthly precipitation with circulation weather types for a dense network of stations over Iberia, Hydrol Earth Syst Sci 17 (2), $2013,665-678$.

Custodio E., Estimation of aquifer recharge by means of atmospheric chloride deposition balance, Contrib Sci 6, 2010, 81 r97. 


\section{elsevier_STOTEN_17069}

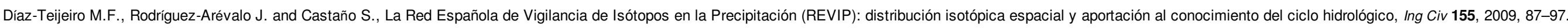

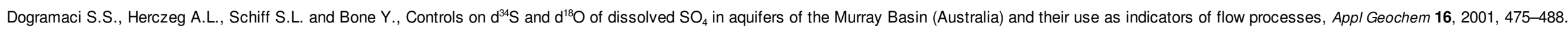

Froehlich K., Kralik M., Papesch W., Rank D., Scheifinger H. and Stichler W., Deuterium excess in precipitation of Alpine regions-_-moisture recycling, Isotopes Environ Health Stud $\mathbf{4 4}$ (1), 2008, 1-10,

http://dx.doi.org/10.1080/10256010801887208.

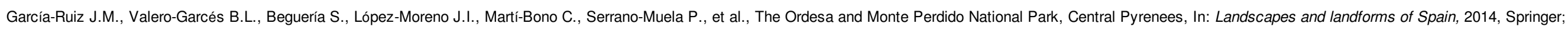

Netherlands, 165-172.

Gonfiantini R., Roche M.A., Olivry J.C., Fontes J.Ch. and Zuppi G.M., The altitude effect on the isotopic composition of tropical rains, Chem Geol 181, 2001, 147-167.

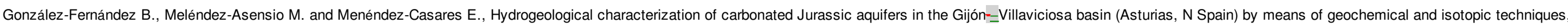
Environ Earth Sci 59 (4), 2009, 913-928.

Goodess C.M. and Palutikof J.P., Development of daily rainfall scenarios for southeast Spain using a circulation-type approach to downscaling, Int J Climatol 18 (10), $1998,1051-1083$.

Gremaud V. and Goldscheider N., Geometry and drainage of a retreating glacier overlying and recharging a karst aquifer, Tsanfleuron-_Sanetsch, Swiss Alps Acta Carso/ 39 (2), 2010, 289-300.

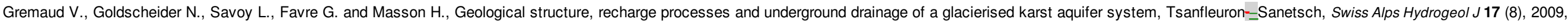
1833-1848.

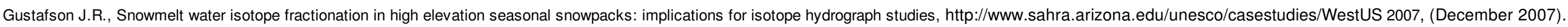

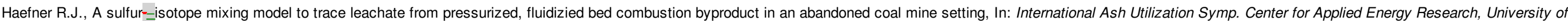
Kentucky, Paper \#280, 1999, [http://www.flyash.info].

Hannah D.M. and McGregor G.R., Evaluating the impact of climate on snow-and ice-melt dynamics in the Taillon basin, French Pyrenees, J Glaciol 43, 1997, 463-568.

Holser W.T. and Kaplan I.R., Isotope geochemistry of sedimentary sulfates, Chem Geol 1, 1966, 93-135.

IGME-OAPN, Guía Geológica del Parque Nacional de Ordesa y Monte Perdido, In: Guías geológicas de Parques Nacionales, 2013, Editorial Everest, (214 pp.).

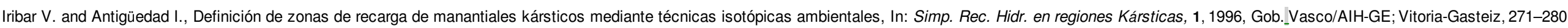

Jenkinson A. and Collison F., An initial climatology of gales over the North Sea, In: Synoptic climatology branch memorandum, 62, 1977.

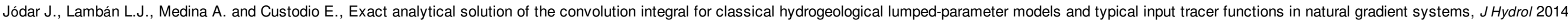
http://dx.doi.org/10.1016/j.jhydrol.2014.10.027.

Jones P.D., Hulme M. and Briffa K.R., A comparison of lamb circulation types with an objective classification scheme, Int J Climatol 13 (6), 1993, 655-663, http://dx.doi.org/10.1002/joc.3370130606.

Kalnay E., Kanamitsu M., Kistler R., Collins W., Deaven D., Gandin L., et al., The NCEP/NCAR 40-year reanalysis project, Bull Am Meteorol Soc 77 (3), 1996,

437-471, [http://dx.doi.org/10.1175/1520-_0477(1996)077 < 0437:TNYRP > 2.0.CO;2].

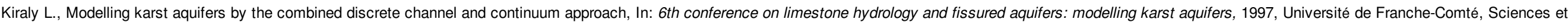
Technique de l:Environnement; La Chaux-de-Fonds, 1-26.

Krouse H.R., Sulphur isotopes in our environment, In: Fritz P. and Fontes J.ChJ.Ch., (Eds.), The terrestrial environment, Isotope Geochemistry vol. 1, 1980 , Elsevier; Amsterdam, 435-471.

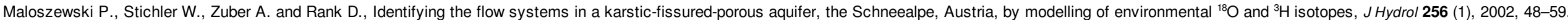




\section{elsevier_STOTEN_17069}

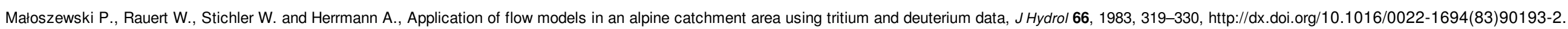
Motyka J., A conceptual model of hydraulic networks in carbonate rocks, illustrated by examples from Poland, Hydrogeol J 6, 1998, 469-482.

Muñoz J.A., Evolution of a continental eollissioncollision belt: ECORS-Pyrenees crustal balanced cross-section, In: McClay K.R., (Ed), Thrust tectonics, 1992, Chapman \& Hall, 235-246.

Muñoz J.A., Martinez A. and Vergas J., Thrust sequences in the Spanish eastern Pyrenees, J Struct Geol 8, 1986, 399-405.

Nriagu J.O. and Coker R.D., Isotopic composition of sulphur in precipitation within the Great Lakes Basin, Tellus 30, 1978, 367-375.

Oerter H. and Ravert W., Core drilling on Vernagtferner (Oetztal Alps, Austria) in 1979: tritium contents, Z Gletscherk Glazialgeol 1982, Volume 18-1, 13-22.

Ohizumi T., Fukuzaki N. and Kusakabe M., Sulfur isotopic view on the sources of sulfur in atmospheric fallout along the coast of the sea of Japan, Atmos Environ 13, 1997, 1339-1348.

Panettiere P., Cortecci G., Dinelli E., Bencini A. and Guidi M., Chemistry and sulfur isotopic composition of precipitation at Bologna, Italy, App/ Geochem 15, 2000, $1455-1467$.

Parish M., A structural interpretation of a section of the Gavarnie nappe and its implications for Pyrenean geology, J Struct Geol 6, 1984, 247-255.

Peel M.C., Finlayson B.L. and McMahon T.A., Updated world map of the Köppen=-Geiger climate classification, Hydrol Earth Syst Sci 11, 2007, 1633-1644, http://dx.doi.org/10.5194/hess-11-1633-2007.

Pérez C., Funcionamiento hidrogeológico de un humedal hipogénico de origen kárstico en las Sierras Marginales Pirenáicas: Las Lagunas de Estaña (Huesca), (PhD. Thesis)2013, Universidad Complutense de Madrid.

Press W.H., Teukolsky S.A., Vetterling W.T. and Flannery B.P., Numerical recipes in C, 1992, Cambridge University Press.

Puig R., Avila A. and Soler A., Sulphur isotopes as tracers of the influence of a coal-fired power plant on a Scots pine forest in Catalonia (NE Spain), Atmos Environ 42 (4), $2008,733-745$.

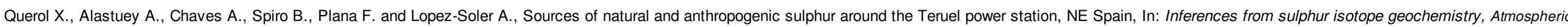
Environment 34, 2000.

Ríos-Aragüés L.M., Introducción al mapa geológico del Parque Nacional de Ordesa y Monte Perdido, In: Sociedad Española de Espeleología y Ciencias del Karst, Boletín 5, 2003, 84-99.

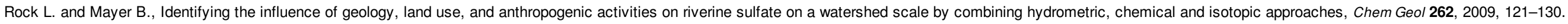

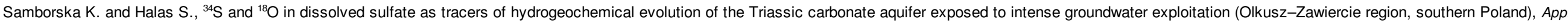
Geochem 25, 2010, 1397-1414, http://dx.doi.org/10.1016/j.apgeochem.2010.06.010.

Sapriza-Azuri G., Jódar J., Carrera J. and Gupta H.V., Stochastic simulation of nonstationary rainfall fields, accounting for seasonality and atmospheric circulation pattern evolution, Math Geosci 45 (5), $2013,621-645$.

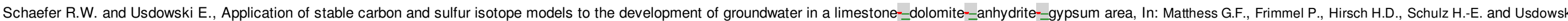
E., (Eds.), Progress in hydrogeochemistry, 1992, Springer; Berlin, 157-163.

Schellart W.P., Analogue modelling of large_scale tectonic_processes: an introduction, $J$ Virtual Explor 7, 2002, 1-6.

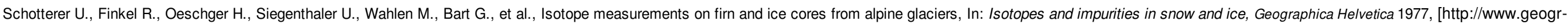
helv.net/68/227/2013/gh-68-227-2013.pdf].

Seguret M., Étude tectonique des nappes et séries décollées de la partie centrale du versant sud des Pyrénées, Ser. Geol. Struct. 2, 1972, Pub. USTELA; Montpellier.

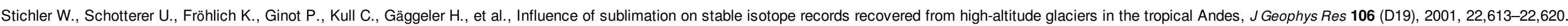

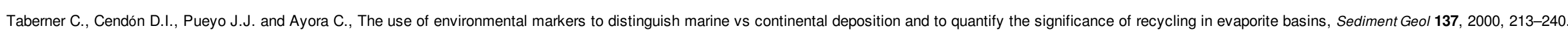

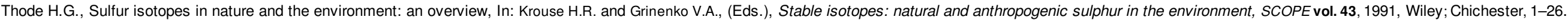




\section{elsevier_STOTEN_17069}

Thode H.G. and Monster J., Sulfur-isotope geochemistry of petroleum, evaporites and ancient seas, In: Young A. and Galley J.E., (Eds.), Fluids in subsurface environments, Am. Ass. Petrol. Geol. Mem.4, 1965, 367-377.

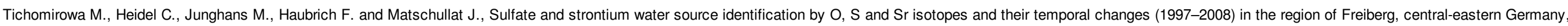
Chem Geol 276, 2010, 104-118.

Van Stempvoort D.R. and Krouse H.R., Controls of ${ }^{18} \mathrm{O}$ in sulfate: review of experimental data and application to specific environments, In: ACS symposium series, American Chemical Society vol. 550, 1994.

Vuille M., Zur raumzeitlichen Dynamik von Schneefall und Ausaperung im Bereich des südlichen Altiplano, Südamerika, Geogr. Bernensia, G45, $1996,1-118$.

Tracer techniques in hydrogeological investigations, In: Zuber A., (Ed), Methodological handbook, 2007, Publishing House of the Wroclaw University of Technology; Wroclaw, [in Polish].

Highlights

\section{- Environmental tracers are essential to study complex alpine karst aquifers}

- The long presence of snow controls the deuterium excess in groundwater.

- Seasonal $\delta \mathrm{D}$ content in springs depends on gap between recharge and discharge points.

- The first hydrogeological characterization of the Ordesa National Park is presented,

- Sulphate content in springs comes from material recycled from Triassic evaporites]

\section{Queries and Answers}

Query:

The term "OMPNP" has been changed to "PNOMP". Please check and amend as necessary.

Answer: OK, PNOMP is the right acronym

Query:

Please provide a definition for the significance of italicized data in Table 2.

Answer: Data which is obtained through a calculation procedure instead of a direct measurement is displayed in the table with an italicized format

Query:

Please identify the corresponding author and provide the correspondence address.

Answer: The corresponding author is J. Jódar

The correspondence address is the following:

Department of Geotechnical Engineering and Geosciences, Technical University of Catalonia (UPC), Barcelona, Spain 


\section{Query:}

The country name "Spain" has been inserted to the 1st and 3rd affiliations. Please check and amend as necessary.

Answer: In both cases it is right.

Query:

The citation "Collins and Young 1981" has been changed to match the author name/date in the reference list. Please check here and in subsequent occurrences, and correct if necessary.

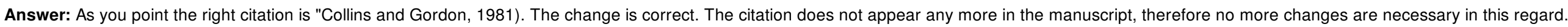
Query:

Please note that Table 4 was not cited in the text. Please check, and correct if necessary.

Answer: You are right. We forgot to cite the Table 4. The point where you have included the citation is not correct. The citation should appear just at the end of the previous paragraph, that is:

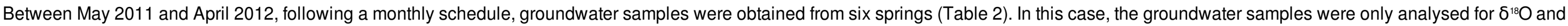
$\delta^{2} \mathrm{H}$ (see Table 4).

Query:

The citation "Iribar et al. (1996)" has been changed to match the author name/date in the reference list. Please check here and in subsequent occurrences, and correct if necessary.

Answer: The change is correct. This citation appears once more in the "Discussion" section but it appears already corrected.

Query:

The citation "Iribar et al. (1996)" has been changed to match the author name/date in the reference list. Please check here and in subsequent occurrences, and correct if necessary.

Answer: The change is correct.

Query:

Uncited references: This section comprises references that occur in the reference list but not in the body of the text. Please position each reference in the text or, alternatively, delete it. Thank you.

Answer: I have deleted the uncited references.

Query:

Please provide the volume number and page range for the bibliography in 'Oerter and Ravert, 1982'.

Answer: Indeed. Volume 18 (1982), Book I, page 13-22

I have included this information in the reference.

Query:

Please confirm that given names and surnames have been identified correctly.

Answer: Yes, all the names have been identified correctly. 


\section{elsevier_STOTEN_17069}

\section{Query:}

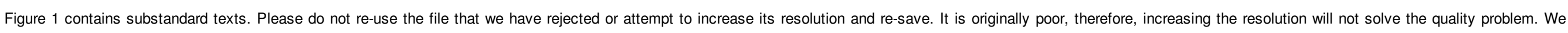

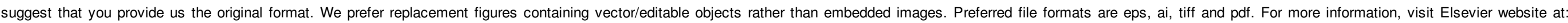
http://www.elsevier.com/author-schemas/artwork-;and-media-instructions/.

Answer: We have attached a new version of Figure 1 with a increased resolution 